\title{
Discrete Element Method Simulations of
}

\section{Bio-Cemented Sands}

\author{
Kai Feng ${ }^{1}$, B. M. Montoya ${ }^{2}$, and T. M. Evans ${ }^{3}$
}

\begin{abstract}
:
Microbially induced calcite precipitation (MICP) has emerged as a novel soil improvement method. In this paper, 3-D discrete element method (DEM) simulations are used to explore the behavior of MICP-cemented sands. Comparisons of the macro-scale response of numerical and physical specimens are made. Microstructure analyses indicate a shear band formed in the numerical specimens, consistent with physical experiments. The bond breakage pattern in numerical specimens is explored and compared to observed measurements from physical specimens. The relationship between dilatancy and stress-strain behavior is evaluated. The results indicate DEM is an effective technique to capture the mechanical behavior of MICP-cemented sand.
\end{abstract}

Keywords: discrete element method; microbial induced calcite precipitation; cemented sand; triaxial tests; shear strength 


\subsection{Introduction}

27 The study of particulate micro-mechanics can provide important insights into the behavior of granular materials. Some microstructure experimental methods, such as resin-impregnation followed by digital image analysis (e.g., Kuo and Frost 1996), magnetic resonance imaging (e.g., Ng and Wang 2001), and X-ray computed tomography (e.g., Wang et al. 2004), have been developed. However, these laboratory tests are difficult to perform, expensive and time consuming. The discrete element method (DEM), which was originally proposed by Cundall and Strack (1979), is an effective simulation technique to provide insight into inter-particle forces and microstructure evolution. Of particular advantage to the current work, specimens with identical initial states can be used for tests under varying cementation levels and confinement. Furthermore, inter-particle information, such as meso-scale void ratio and coordination number, is accessible during any stage of the simulation.

Using DEM, many researchers have explored the behavior of clean sand under biaxial tests (e.g., Iwashita and Oda 2000), triaxial tests (e.g., Cui et al. 2007; Belheine et al. 2009), direct shear tests (e.g., Ni et al. 2000; Jacobson et al. 2007; Zhang and Thornton 2007), and plain strain tests (Suiker and Fleck 2004). Particularly, Zhao and Evans (2009) compared the mechanical properties of granular soils under triaxial, plane strain and direct shear loading states and concluded that DEM is capable of simulating varying loading conditions successfully without model recalibration.

Cementation, in either natural or artificial form, exists as an important improvement medium to strengthen soil static properties (e.g., Clough et al. 1981; Airey 1993; Huang and Airey 1998;

47 Ismail et al. 2002; Chiu et al. 2009) and dynamic properties (e.g., Saxena et al. 1988; Clough et al. 1989). Nevertheless, traditional artificial cementation media, such as Portland cement and gypsum, have limited injection distance due to high viscosity (Struble and Sun 1995). Recently, microbial induced calcite precipitation (MICP) has emerged as a new ground improvement strategy given its advantages of longer treatment distances and environmentally innocuous 
approach (DeJong et al. 2013). Taking advantage of urea hydrolysis catalyzed by a common soil bacteria Sporosarcina pasteurii, carbonate precipitation is created at soil grain contacts (DeJong et al. 2006; Mortensen et al. 2011; Burbank et al. 2013). The improvement on soil properties through MICP has been verified by soil column tests (e.g., Whiffin et al. 2007; Martinez et al. 2013), unconfined compressive tests (e.g., Qabany and Soga 2013; Shanahan and Montoya 2014), 1-D compression tests (e.g., Feng and Montoya 2014, Lin et al. 2016), triaxial tests (e.g., DeJong et al. 2006; Montoya and DeJong 2015; Feng and Montoya 2015), large scale laboratory tests (e.g., van Paassen et al. 2010) and in situ cone penetration test (e.g., Burbank et al. 2013). Fauriel and Laloui (2012) proposed a bio-chemo-hydro-mechanical model for bio-soil grouting application and made corresponding numerical examples. Although their model provides a good continuum solution to the coupled treatment in MICP processes, limited investigation is made on the mechanical response variation derived from MICP cementation.

Scanning electron microscopy (SEM) is able to provide evidence on the existence of calcite between soil particles when the specimen is dissected for analysis after shearing (DeJong et al. 2010); however, to the authors' knowledge, no insight can be obtained on the microstructure evolution and bond breakage mechanism of MICP cemented soil under shearing, which is important to understand the macro-response of cemented soil. Using X-ray tomography, Tagliaferri et al. (2011) observed localized deformation of MICP cemented sands during shearing and inferred cementation breakage pattern; while important, that work provides only qualitative information on the evolution of bond breakage rather than a robust quantitative analysis. Thus, it is beneficial to use DEM models to improve our understanding of the behavior and better inform the engineered design of bio-cemented sand, because these models can supply both qualitative and quantitative information on microstructure.

Furthermore, as mentioned above, although DEM of unbonded particles have been conducted by many previous researchers, the exploration on cemented sand is more limited (e.g., Wang and Leung 2008; Estrada et al. 2010, Evans and Ning 2013; de Bono et al. 2014a,b; Ning et al. in review), and always focused on chemical, rather than bio-mediated, cementation. Although an enhanced understanding of the mechanical behavior of cemented soil was provided by this 
prior work, it is not obvious that these results are applicable to bio-cemented sands, particularly when they are subjected to the full range of loading conditions applied in laboratory and field testing. A new bonding approach specifically tailored to DEM simulations of bio-cemented soils has recently been proposed (Evans et al. 2014; Khoubani et al. 2016), but this work is exploratory in nature and presently confined to simulation of relatively small particle assemblies.

In the current study, the response of MICP cemented sands from physical experiments are summarized, followed by the numerical model calibration corresponding to strength and deformation characteristics from physical experiments. Macro-response characteristics of the numerical specimens, and global void ratio and coordination number analyses are presented. Associated microstructure properties, such as mesoscale void ratio and coordination number, are analyzed in statistical and contour slices forms. Bond breakage patterns and evolution are captured for cemented physical specimens and related to the microstructure variation of the numerical specimens. Particularly, the bond breakage pattern evolution from the numerical model is compared to the bond breakage pattern from physical specimens, which is estimated using shear wave velocity measurements. The dilatancy characteristics and effect of cementation on soil behavior is discussed in detail.

\subsection{Experimental Procedure and Results}

The experimental MICP treatment process and the mechanical response of bio-cemented sands are summarized below. Traditional cementation media, such as Portland cement or gypsum, are normally applied by dry mixture with soil in the laboratory (e.g., Ismail et al. 2002). MICP, which has a similar process to natural deposition (Morad 1998), is gradually precipitated onto the sands grains and at the sand particle contacts. Ottawa 50-70 sand was selected as the sand matrix in this study. Index properties are summarized in Table 1.

Sporosarcina pasteurii (American Type Culture Collection, ATCC 11859) was the biological organism used to induce urea hydrolysis. To grow the bacteria to the desired population 
density, an ammonium-yeast extract media (ATCC 1376) was used. The growth media was inoculated with the S. pasteurii stock culture aerobically at $30{ }^{\circ} \mathrm{C}$ in a shaking incubator at 200 rpm for approximately 40 hours before harvesting. Suspended culture after inoculation was then centrifuged at $4000 \mathrm{~g}$ for 15 minutes. The supernatant was removed after the centrifuge period and replaced with fresh growth media. Finally, the desired bacteria were stored in the centrifuge vials at $4^{\circ} \mathrm{C}$ until used.

A two-phase injection procedure was used to induce cementation: in the first stage, suspended bacteria was injected through the specimen and retained in the pore space for at least six hours allowing for bacteria attachment; in the second stage, cementation media were repeatedly injected until the desired level of cementation was reached. A summary of chemical components and concentrations for the biological and chemical media is presented in Table 2 .

Soil specimens were prepared by dry pluviation to a state with a similar initial global void ratio, approximately e $=0.72$ (loose). To explore the effect of density, dense (e $=0.63$ ) and medium dense $(e=0.70)$ specimens were also prepared. Complete details of the physical experiments can be found in Feng and Montoya (2015). Cemented samples were then confined under varying pressures $(100 \mathrm{kPa}, 200 \mathrm{kPa}$, and $400 \mathrm{kPa})$ and injected with cementation media to reach the target cementation levels. Once the cementation process was completed, backpressure saturation was conducted in order to obtain a B value greater than 0.95 . The cementation content of specimens was finally determined by the acid washing method, after shearing under a rate of $2.5 \%$ per hour to a maximum axial strain of $25 \%$.

Representative experimental results at a confinement of $100 \mathrm{kPa}$ were selected to calibrate the numerical model. The experimental uncemented loose sand exhibits slightly dilative behavior, with shear stiffness, peak strength, strain-softening tendencies, and dilative volumetric strains increase with increasing density (Figure 1). The experimental MICP cemented loose sand demonstrates a significant increase in initial stiffness, peak strength, and dilative volumetric strains compared to the loose uncemented sand (Figure 2). Additionally, the peak strength of the MICP cemented specimens increases with an increase in cementation level. The experimental behavior is further presented in subsequent sections of the manuscript for 
comparisons to the simulated behavior. Detailed discussions of the experimental behavior can be found in Feng and Montoya (2015).

\subsection{Model and Material Properties}

For comparison to laboratory results, three-dimensional DEM simulations were performed using PFC $^{3 D}$ (Itasca 2008). Axisymmetric compression element tests were simulated on 2:1 H:D cylindrical assemblies of approximately 7,000 particles with material properties similar to those of a clean quartz sand. The numerical model was first calibrated to uncemented sand behavior, as discussed subsequently. Initial conditions, boundary conditions, and model scaling were viewed to be of particular importance to the simulations and were considered in detail. Material and model properties are discussed in the following paragraphs and summarized in Table 3.

DEM simulations are typically comprised of two geometric entities: balls and walls. This makes the simulation of flexible membrane confinement, such as that used in physical triaxial experiments, challenging. Previous researchers have used strings of balls linked by contact bonds to simulate membranes in two dimensional simulations (e.g., Evans and Frost 2008; Wang and Leung 2008; Evans and Frost 2010). A similar mechanism could be used in three dimensional models, but it increases the computational time significantly and is complex to implement. In the current study, stacks of cylindrical walls are used to simulate the flexible membrane (Zhao and Evans 2009), which saves computational time and allows the numerical membrane to deform independently since walls in $P F C^{3 D}$ do not interact with each other. Due to the frictional end platens in physical experiments, friction was also applied to top and bottom platens in the numerical specimens.

The maximum stable timestep for the calculation is related to the minimum particle mass in the assembly. The simulation time could be several months if actual particle sizes were used. To increase calculation efficiency, it is necessary to scale the numerical soil particles so that the maximum timestep can be increased. Generally, the three primary methods for model scaling 
are density scaling (e.g., Thornton 2000; Cui and O'Sullivan 2006), gravity scaling (e.g., Jiang et al. 2007) and mass scaling (e.g., Jacobson et al. 2007; Belheine et al. 2009; Evans and Valdes 2011). In the current study, mass scaling was implemented to improve calculation efficiency. The grain size distribution (GSD) of real particles and numerical particles are shown in Figure 3, from which we can see that the GSD of real particles and numerical particles are the same but numerical grains have a diameter in 1000 times larger than real particles. The specimen size was scaled up accordingly. Note that the dimensions of numerical model remain consistent with physical experiments: a 2:1 height:diameter ratio.

Spherical particles tend to overestimate the rotations of soil grains, as first observed by Bardet (1994). To overcome this problem, each particle in the numerical simulations is comprised of two identical overlapping spheres clumped together such that the aspect ratio of the overall particle is 1.5:1 (Figure 4a). Therefore, each assembly of approximately 7,000 grains was comprised of approximately 14,000 spherical particles.

Numerical assemblies were prepared by generating particles with random diameters and random orientations at random locations within the specimen volume. All random numbers were selected from a uniform distribution $\in[0,1]$ and mapped to the desired range of magnitudes. Because particle locations were only hard-core random (i.e., at machine precision, no two particles can have the same centroid), the original assemblies contain large particle overlaps (contact forces). Thus, samples were eased to an equilibrium state by stepping forward through time while zeroing the assembly's kinetic energy every few calculation steps to prevent large accelerations.

After the numerical specimens were initially equilibrated, they were isotropically consolidated to a mean effective stress of $100 \mathrm{kPa}$. The as-consolidated void ratio was controlled by varying the initial friction coefficients of the particles; values of $0.05,0.10$, and 0.17 were used for the dense, medium, and loose specimens, respectively (i.e., a higher friction coefficient during consolidation will result in a looser equilibrated assembly because the friction frustrates translational motion). The consolidated specimens were axially compressed at a strain rate of 
$1.25 \times 10^{-3} / \mathrm{s}$, corresponding to an inertial number of $I=4 \times 10^{-5}$, consistent with quasistatic deformations (i.e., $I<10^{-3}$ ).

191 For calibration, the numerical simulations were compared to the stress-strain-volume change response measured in physical experiments of uncemented sand, as shown in Figure 1. Particle stiffness and friction coefficient were adjusted so that specimen-scale numerical response most closely agreed with the macroscale results from physical experiments. The material parameters obtained through this procedure (Table 3) are still consistent with the known physical 196 properties of quartz sand grains and with the values adopted by previous studies of Ottawa sand (e.g., Zhao and Evans 2009). The numerical model captures the physical responses in 198 general, although it tends to overestimate the volumetric deformation and underestimate residual strength. The overestimation of dilatant tendencies is typical in DEM simulations because the specimen boundary conditions are not as compliant as a latex membrane. The denser the initial soil state is, the higher the peak strength and dilative deformation are obtained. Detailed analysis about initial, peak and residual state will be discussed later.

Parallel bonds were used to simulate cementation between particles (Potyondy and Cundall 2004). A parallel bond is a cylinder of material between two particles in contact, as shown in Figure 4a. This arrangement is close to the real formation of MICP as captured by the microscopic images shown in Figures $4 \mathrm{~b}$ and 4c. Parallel bonds resist shear, tension, and compression (the latter in parallel with the particles' contact stiffness) and transmit moment. The parameters and their corresponding values used to define the parallel bonds are presented in Table 3. These values were determined through calibration to a series of physical experiments, as discussed subsequently. To simulate different cement contents, different values of the bond radius parameter were used. This parameter controls the theoretical volume 212 of cement applied to the assembly by defining the cross-sectional area of the bond connecting 213 any two particles (i.e., a lower bond radius corresponds to a lower cement content). Similar to 214 the cemented physical specimens, parallel bonds in the numerical simulation experience 215 progressive breakage during loading when the shear or normal forces on them exceed their 216 shear or normal strength. In physical specimens the bond breakage can occur as a cohesive 
217 failure (bond breakage within the calcite phase) and as an adhesive failure (bond breakage 218 between the calcite minerals and sand grains). The simulation does not distinguish between 219 cohesive and adhesive failures; instead, the parallel bond fails whenever the load carrying 220 capacity is exceeded at a given contact. In the numerical model, it is possible to record the bond 221 breakage events and corresponding bond information when the bonds are broken, and analyze 222 the bond breakage pattern.

223 Calibration of the numerical model for MICP cemented sand was performed by using the uncemented soil properties and calibrating the bond properties (e.g., stiffness, strength, radius)

225 for the heavily cemented specimen only. The lightly and moderately cemented specimens were 226 simulated by reducing the bond radii accordingly to match the mechanical behavior from 227 experimental specimens. This is consistent with our understanding (Feng and Montoya 2015) 228 that the degree of cementation varies specifically with the amount of cement per unit volume 229 deposited at particle contacts. Stress-strain-volume change response of the numerical and 230 physical experiments for MICP cemented sand are presented in Figure 2. The numerical model 231 captures the effect of cementation levels on soil behavior: soils with higher cementation level 232 present larger strength and dilative deformation; cemented soil shows brittle behavior after 233 peak strength and this brittle behavior increases with the increase of cementation contents, 234 which comply with the experimental results. The simulated results underestimate the residual 235 strength of physical samples because the bond model implemented herein does not consider 236 the densification effect from degraded cementation. With regard to volumetric deformation, 237 physical specimens present a faster initial dilation rate but smaller final dilative deformation.

\subsection{Simulation Results}

\subsection{Macro-response}

241 To assess the ability of the numerical simulations to capture the observed response from 242 physical experiments, the initial stiffnesses, peak strengths, and residual strengths are 
compared in Figure 5. The initial tangent modulus was used to represent initial stiffness and residual strength was chosen as the mobilized friction angle at $15 \%$ axial strain.

Figure $5 \mathrm{a}$ indicates that the initial stiffness of uncemented soil was captured very well by the numerical model while the initial modulus of cemented soil with light and moderate cementation levels was underestimated. Since it was used to calibrate the numerical model, the initial modulus of the heavily cemented numerical sample was consistent with the initial modulus of the physical sample. The relatively higher initial modulus for the experimental lightly and moderately cemented specimens is likely related to the laboratory MICP process (e.g., combined void filling and particle bonding). The numerical model presented in the current study only changes the parallel bond radius to represent changes in the cementation level.

Figures $5 b$ and $5 c$ summarize the comparison of peak and residual strength. As mentioned above, the numerical model predicts peak strength very well, but underestimates residual strength. Particularly, the numerical samples are all about to reach a similar residual strength at $15 \%$ axial strain, regardless of initial density or cementation level.

In summary, the current numerical model generally captures the mechanical behavior of the physical experiments. Appropriate trends for the strength, stiffness, and volumetric strain are observed with the increase in cementation level. The proposed DEM model also provides a method to investigate the micro-response of MICP cemented soil, as discussed in the following section.

\subsection{Micro-response}

Macro-response is always connected to the micro-response of the soil element. One clear example is the formation of a shear band in uncemented dense or cemented sand, which is driven by microscale processes but governs engineering-scale behavior (Evans and Frost 2010). Using DEM simulations, it is possible to analyze the state of granular assemblies at the microscale using accessible parameters, such as global void ratio, mesoscale void ratio, and 
coordination number. Furthermore, corresponding contour plots of the soil properties provide insight into the spatial variability of these properties within the specimen.

Global void ratio is the average void ratio for the entire specimen, which can reflect the general void space evolution. In Figure 6, the evolution of global void ratio for six specimens is presented. Note that the decrease in void ratio due to the void filling effect of the MICP cementation is not considered. As expected, the medium dense and dense specimens demonstrate lower initial void ratio and other specimens have the same initial void ratio due to their identical initial states. At large strains, the uncemented loose and medium dense specimens and cemented specimens with light and moderate cementation levels were sheared to a similar final void ratio. The uncemented dense specimen dilated, but not to as loose of a condition as the other uncemented specimens. This behavior is likely due to the denser initial condition, which is consistent with the "terminal densities" observed by Narsilio and Santamarina (2008). The heavily cemented specimen terminated at the lowest void ratio due to the highly localized shearing zone at large strains. Evans and Frost (2010) showed that in both physical and numerical specimens, dilation outside of the shear band ceased after the onset of localized deformation.

The localization is illustrated in Figure 7, where the mesoscale void ratio are plotted at $0 \%, 1 \%$, $5 \%, 10 \%$ and $15 \%$ axial strain for seven specimens with different initial void ratio, cementation levels, or confinement. For the uncemented dense specimen, it is observed from Figure $7 \mathrm{c}$ that a localization zone starts to form at the middle of the sample height at about $10 \%$ strain, consistent with shear band formation observed in physical experiments. There is not a clear shear band in the uncemented medium dense (Figure 7b) and loose specimens (Figure 7a) at 10\% axial strain. Nevertheless, uncemented loose and medium dense specimens present a localization zone at $15 \%$ strain, which is not supported by physical experiments at similar strain levels. However, if the physical specimens are sheared further to larger axial strain (e.g., 25\%), a shearing band is also expected to show in both loose and medium dense specimens. Both lightly (Figure $7 \mathrm{~d}$ ) and moderately (Figure 7e) cemented samples tend to present a localization zone at $15 \%$ strain while a clearer and looser localization zone occurs to heavily cemented 
specimen (Figure $7 f$ ) at around $10 \%$ stain. The thickness of localization zone from heavily cemented specimen is observed to be thinner than that of specimens with lower cementation contents; this trend was observed in the physical experiments as well. The seventh specimen is a heavily cemented sample sheared under $400 \mathrm{kPa}$ confinement (Figure $7 \mathrm{~g}$ ). Comparing the heavily cemented specimens sheared under different confinements (Figure $7 \mathrm{f}$ and $7 \mathrm{~g}$ ), the higher level of confinement tends to suppress the specimen from dilating and constrain the formation of a shear band, which is consistent with the volumetric deformation observed from the macro-response of the physical experiments (Feng and Montoya 2015).

To summarize, three points can be made in regards to the shearing band formation: 1) for uncemented soil, specimens with denser states tend to present a shear band earlier compared to specimens with looser states; 2) for cemented soil, specimen with higher cementation levels are prone to show a clearer and thinner shear band at earlier strain levels compared to specimens with lower cementation levels; and 3) higher levels of confinement have a tendency to constrain the development of a shear band.

The coordination number $(c n)$ is the average number of contacts per particle for the whole assembly. Evolution of $c n$ with strain for each of the simulations is presented in Figure 8 . The dense uncemented specimen has an initial coordination number of 7.8 , which is the highest among all of the simulations. The medium dense uncemented soil has an initial coordination number of 7, which reflects its denser soil state compared to the loose uncemented specimen that has an initial coordination number of 6 . Since all the cemented specimens have an identical initial fabric as that of the loose uncemented specimen, they possess the same initial coordination number. During shearing, all of samples demonstrate a continuous decrease in coordination number until they approach an axial strain of $15 \%$. There is an abrupt reduction in coordination number within $1 \%$ strain for all the specimens, after which the trend becomes relatively flat. In contrary, there are no significant changes in the void ratio contour plots (Figure 7) during the first $1 \%$ of global axial strain. These results imply that particle rearrangement begins early in the shearing process without significant changes in mesoscale void ratio, after which the assembly begins to dilate. 


\subsection{Bond breakage analysis}

327 Cementation changes the geometric distribution of the forces transmitted through a granular 328 assembly. Under shearing, the cemented bonds will progressively break. In physical experiments, cemented soils can be monitored using shear wave velocity which can provide information on bond breakage during shear (e.g., Montoya and DeJong 2015); however, it is difficult to quantify the bond breakage pattern or evolution in the laboratory. Quantitative bond breakage analysis is feasible using DEM. In the current work, bond breakage is explored through statistic and visualization analyses. Furthermore, the relationship between the bond breakage pattern and soil mechanical response is also investigated.

As mentioned previously, it is feasible to evaluate the bond breakage pattern by monitoring shear wave velocity $\left(V_{s}\right)$ in physical experiments, although it is an indirect measurement of bond breakage. In order to estimate the evolution of bond breakage during shearing for a physical specimen, the $V_{S}$ data during shear for a moderately cemented specimen subjected to drained triaxial compression was used (Montoya and DeJong 2015). In order to correlate change in $V_{S}$ to cement bond breakage, the difference in shear wave velocity before and after MICP treatment is selected to represent the reference value for intact cemented specimens and the changes in shear wave velocity during shearing are correlated to the fraction of broken bonds. In the bond breakage estimation, it is assumed that the change of shear wave velocity is only due to the breakage of cementation. This assumption tends to underestimate the breakage percentage of cementation bonds since the changes in $V_{s}$ are due to the combined effects of the increase in $V_{s}$ from increasing mean effective stress and the decrease in $V_{s}$ from bond breakage. Bond 347 breakage as a function of global axial strain is shown in Figure 9 for both physical and numerical specimens. Numerical results demonstrate quite similar bond breakage pattern to the estimation from physical experiments, though the numerical specimens present higher breakage percentage compared to the physical trend with a similar cementation level.

351 As previously discussed, $V_{s}$ is also affected by the mean effective stress in a particulate assembly, which means that using the recorded shear wave velocity to estimate bond breakage 
353 results in an underestimation of bond breakage. Therefore, the estimation of bond breakage 354 from physical experiments can be modified by using normalized shear modulus (G) instead of 355 shear wave velocity $\left(V_{S}\right)$. In order to remove the effect from varying mean effective stress, the 356 shear modulus was normalized by $\sqrt{p^{\prime} p_{a t m}}$ where $p^{\prime}=$ mean effective stress and $p_{a t m}=$ 357 atmospheric pressure $(101.3 \mathrm{kPa})$ because shear modulus is proportional to the square root of 358 mean effective stress (Hardin and Drnevich 1972; Stokoe et al. 1985). Based on this logic, a 359 modified bond breakage pattern was plotted according to the modified shear modulus. The 360 bond breakage pattern from the new estimation is quite similar to the calculation from numerical specimen with moderate cementation level, which is also the cementation range reported by Montoya and DeJong (2015). The relationship between shear wave velocity of the physical specimen and estimated bond breakage percentage is illustrated in Figure 10. The 364 relationship between $V_{s}$ and the estimated fraction of broken bonds is approximately parabolic. However, due to the multiple interacting factors that control shear wave velocity during shearing (e.g., mean effective stress, cementation level, coordination number, stress anisotropy), the relationship between $V_{s}$ and the fraction of broken bonds may not be unique.

During loading, specimen deformation is constrained by lateral confinement and different 369 levels of confining pressure may cause varying volumetric deformation characteristics and thus affect the bond breakage pattern. To investigate this point, the bond breakage pattern of heavily cemented numerical specimens under varying confinements is shown in Figure 11. 372 Higher confinement (e.g., $400 \mathrm{kPa}$ ) leads to more cementation breakage. At around 10\% axial 373 strain, the bond breakage percentage for the specimen under $400 \mathrm{kPa}$ confining pressure is 374 close to $100 \%$. A shear band still occurs within the heavily cemented specimen with $400 \mathrm{kPa}$ confinement from the mesoscale void ratio contours presented in Figure $7 \mathrm{~g}$. A shear band was 376 also observed from physical experiments under the same cementation level and confinement. 377 The densification effect from calcite cementation is considered to explain the formation of 378 shearing band in associated physical specimens. There is no densification effect in the numerical specimens, which implies that the microstructure evolution was still affected by the existence of bonds, leading to shear banding at high global axial strains. 
381 The coordinate projections of intact and broken bonds for the heavily cemented specimen 382 sheared under $100 \mathrm{kPa}$ confinement are plotted in central vertical plane at 1\% (Figure 12 a) and $38310 \%$ (Figure 12 b) axial strain. Note that only the bonds locating within 0.1 unit distance to the central vertical plane were chosen to make the plot. In this figure, the red color represents bonds in intact conditions while blue color indicates broken bonds. It is clearly concluded that the broken bonds concentrate in the highly localization zone, as shown in mesoscale void ratio contours (Figure 7). Outside the localization zone, there are few broken bonds, which was qualitatively verified with SEM images (Montoya and Feng 2015).

The effect of the evolution of bond breakage on the mechanical behavior of an MICP-cemented 390 specimen is explored in Figure 13. The heavily cemented specimen sheared under $100 \mathrm{kPa}$ confinement is shown for illustration. Specimen response from $0 \%-1 \%$ global axial strain is highlighted in the figure inset, which implies that only a small portion of bonds are broken before peak strength and an elastic response is maintained. After peak strength, the specimen experiences dramatic bond breakage events and subsequently, the specimen experiences gradual bond breakage until $15 \%$ strain, at which point a stabilized constant rate of bond breakage is predicted to occur. In other words, for MICP cemented sands, intensive bond breakage events occur as peak strength is obtained. In contrast, findings from Wang and Leung (2008) indicated that dramatic bond breakage events occurred to Portland cement treated sands at yield, which is the earlier than peak strength. The extremely high initial stiffness of 400 MICP cemented soil moves the yield stress very close to the peak stress and may help explain the differences between MICP and Portland cement treated soil.

402 The bond breakage pattern is also related to the variation of coordination number, which 403 characterizes the packing structure of granular assemblies. Figures 9 and 11 demonstrate that 404 the bond breakage rate is highest during the first 1\% of global axial strain; at the same time, the 405 coordination number of the specimen reduces significantly (Figures 8 and 14), indicating the 406 consistency between bond breakage pattern and global loss of interparticle contacts. After the 407 initial rapid changes (e.g., after 1\% strain), the coordination number is essentially constant at a 408 value of approximately $c n=5$, which may demonstrate the formation of major unbonded force 
chains as the primary load-bearing mechanism for the assembly; the bond breakage trend also tends to be more gradual with about $30 \%$ cementation bonds still breaking between $1 \%$ and $15 \%$ strain, which may come from the ongoing fabric evolution in the highly localized shear zone.

\subsection{Dilatancy and Strength}

The shear resistance of sand comes from both friction, which is discussed above, and dilatancy, which is discussed here. The uncemented loose and dense specimens and heavily cemented specimens with $100 \mathrm{kPa}$ confinement are selected to analyze stress dilatancy (Figures 15-17).

Dilatancy is defined as $-\delta \varepsilon_{v}^{p} / \delta \varepsilon_{s}^{p}$ (Wang and Leung 2008), where $\delta \varepsilon_{v}^{p}$ is the increment of plastic volumetric strain and $\delta \varepsilon_{s}^{p}$ is the increment of plastic shear strain. Numerical and experimental specimens are analyzed side by side.

For uncemented sand, numerical specimens tend to present larger dilatancy compared to experimental specimens (Figures 15 and 16); those results are consistent with the higher dilative volumetric deformation and faster dilative rate for numerical model demonstrated in Figure 1b. For both numerical and physical specimens, the axial strain at the peak strength is very close to the axial strain at the maximum dilatancy. However, for heavily cemented specimen, the maximum dilatancy happened at an axial strain larger than that for peak strength (Figure 17), which agrees with the two-dimensional numerical findings from Wang and Leung (2008), in which they found a delayed maximum dilatancy development for Portland cement treated samples. This response is related to the suppression of dilatancy before yielding due to the presence of cementation, which is contrary to the behavior of purely frictional material (Leroueil and Vaughan 1990), as demonstrated by the uncemented specimen reference behavior.

\subsection{Limitations of current model}


434 In the model presented herein, the cementation was captured using parallel bonds, which 435 simulate the shape and distribution of experimental cementation very well. However, in the 436 current simulation system, the bond will disappear when the contact forces exceed its shear or 437 normal strength, which does not comply with the physical reality. In triaxial testing, it is 438 expected the cementation particles will crush during shearing and become calcite fines 439 between the original soil particles, or part of cementation particles will remain attached to the 440 soil grains even when the cementation bonds were broken (DeJong et al. 2010). Using the 441 current model, residual strength is underestimated and the corresponding microstructure may 442 not exactly reflect the physical specimen, although qualitative microstructure evolution is 443 captured. Therefore, a model considering the post-broken effect of cementation is needed, and 444 further investigations are ongoing (e.g., Evans et al. 2014; Khoubani et al. 2016).

445 In the current model, both cementation size and cementation distribution were assumed as 446 uniform, which is not a real case in physical experiments (Feng and Montoya 2015). It is worthy 447 of further explorations on the bond distribution effect to the response of MICP cemented sand 448 in both macro- and microscales.

449 The formation of localization zone was successfully predicted with the current numerical model; 450 however, the inclination of the shear band was inconsistent with observations from physical 451 specimens. The end restraint or dead zone in the top and bottom boundary explains the 452 occurrence of shearing band in physical triaxial tests without lubricated ends (Lee 1978). 453 Although platen friction was added in current model, it was not sufficient to produce the same 454 effect as that in physical experiments. Future study on the boundary effect would be necessary.

$456 \quad 6.0 \quad$ Summary and Conclusions

457 A discrete element numerical model for MICP cemented soil was explored and the following 458 conclusions can be made: 
1. The proposed model captured the initial stiffness and peak strength of uncemented soil well, but it underestimates residual strength. For cemented soil, the peak response was predicted correctly by numerical model, although the model underestimates both initial stiffness and residual strength, which can be explained by the limitations of current cementation model;

2. The formation of the shear band was captured through the meso-scale void ratio contours in the current model, although the degree of inclination was not predicted successfully, which is likely due to different boundary conditions between experimental and numerical specimens; in physical specimens, the formation of shearing band is considered to be related to the densification effect from calcite cementation. In numerical specimens, densification effect is not considered and shear banding at high global axial strains was affected by the existence of bonds;

3. Using the numerical model, statistical and 3-D slices visualization analysis of the bond breakage pattern are made. The bond breakage pattern from numerical specimen is similar to that estimated from physical specimen with shear wave velocity monitoring. The intact and broken bonds can be visualized using recorded bond information, which implies that bond breakage is concentrating in a localized zone formed during shearing; and

4. The numerical model presents the similar pattern of dilative response compared to experimental data. Due to the cemented structure created by MICP cementation, both numerical and physical cemented specimens demonstrate delayed maximum dilatancy after peak strength. In contrast, for uncemented specimens, peak strength and maximum dilatancy occur at the same axial strain.

In summary, it is shown that the DEM is capable of reasonably simulating the macro- and microresponses of uncemented and MICP cemented soil; a well-calibrated DEM model is expected to be useful to predict the mechanical properties of MICP cemented soil and provide insight in the engineering design. 
Funding from the National Science Foundation (CMMI \#1537007 \& 1538460) is appreciated. Any opinions, findings, and conclusions or recommendations expressed in this material are those of the authors and do not necessarily reflect the views of the National Science Foundation. This work was performed in part at the Analytical Instrumentation Facility (AIF) at North Carolina State University, which is supported by the State of North Carolina and the National Science Foundation (ECCS \#1542015). The AIF is a member of the North Carolina Research Triangle Nanotechnology Network (RTNN), a site in the National Nanotechnology Coordinated Infrastructure (NNCl).

\section{References}

Airey, D. W. (1993). "Triaxial testing of naturally cemented carbonate soil." J.Geotech.Engrg., 119(9), 1379-1398.

Bardet, J. P. (1994). "Observations on the effects of particle rotations on the failure of idealized granular materials." Mech.Mater., 18(2), 159-182.

Belheine, N., Plassiard, J. P., Donze, F. V., Darve, F., and Seridi, A. (2009). "Numerical simulation of drained triaxial test using $3 \mathrm{~d}$ discrete element modelling." Computers and Geotechnics, 36(1-2), 320-331.

Burbank, M., Weaver, T., Lewis, R., Williams, T., Williams, B., and Crawford, R. (2013). "Geotechnical tests of sands following bioinduced calcite precipitation catalyzed by indigenous bacteria." J.Geotech.Geoenviron.Eng., 139(6), 928-936.

Chiu, C. F., Zhu, W., and Zhang, C. L. (2009). "Yielding and shear behaviour of cement-treated dredged materials." Eng.Geol., 103(1-2), 1-12.

Clough, G. W., Sitar, N., Bachus, R. C., and Rad, N. S. (1981). "Cemented sands under static loading." Journal of Geotechnical and Geoenvironmental Engineering, 107(GT6), 799-817. 
513 Clough, G., Iwabuchi, J., Rad, N., and Kuppusamy, T. (1989). "Influence of cementation on liquefaction of sands." J.Geotech.Engrg., 115(8), 1102-1117.

515

Cui, L., and O'Sullivan, C. (2006). "Exploring the macro- and micro-scale response of an idealised granular material in the direct shear apparatus." Géotechnique, 56(7), 455-468.

Cui, L., O'Sullivan, C., and O'Neill, S. (2007). "An analysis of the triaxial apparatus using a mixed boundary three-dimensional discrete element model." Géotechnique, 57(10), 831-844.

Cundall, P. A., and Strack, O. D. L. (1979). "A discrete numerical model for granular assemblies." Géotechnique, 29(1), 47-65.

de Bono, J.P., and McDowell, G.R. (2014). "DEM of triaxial tests on crushable sand." Granular Matter, 16, 551-562.

de Bono, J.P., McDowell, G.R., and Wanatowski, D. (2014). "DEM of triaxial tests on crushable cemented sand." Granular Matter, 16, 563-572.

DeJong, J. T., Soga, K., Kavazanjian, E., Burns, S., van Paassen, L. A., Qabany, A. A., Aydilek, A., Bang, S. S., Burbank, M., Caslake, L. F., Chen, C. Y., Cheng, X., Chu, J., Ciurli, S., Esnault-Filet, A., Fauriel, S., Hamdan, N., Hata, T., Inagaki, Y., Jefferis, S., Kuo, M., Laloui, L., Larrahondo, J., Laloui, L., Martinez, B., Montoya, B. M., Nelson, D. C., Palomino, A., Renforth, P., Santamarina, J. C., Seagren, E. A., Tanyu, B., Tsesarsky, M., and Weaver, T. (2013). "Biogeochemical processes and geotechnical applications: progress, opportunities and challenges." Géotechnique, 63(4), 287-301.

DeJong, J. T., Fritzges, M. B., and Nusslein, K. (2006). "Microbially induced cementation to control sand response to undrained shear." J.Geotech.Geoenviron.Eng., 132(11), 1381-1392.

DeJong, J. T., Mortensen, B. M., Martinez, B. C., and Nelson, D. C. (2010). "Biomediated soil improvement." Ecol.Eng., 36(2), 197-210.

Estrada, N., Lizcano, A., and Taboada, A. (2010). "Simulation of cemented granular materials. II. Micromechanical description and strength mobilization at the onset of macroscopic yielding." Phys Rev E., 82(1), 011304. 
543

544

545

546

547

548

549

550

551

552

553

554

555

556

557

558

559

560

561

562

563

564

565

566

567

568

569

570

571

Evans, T. M., and Frost, J. D. (2008). "Membrane effects on microstructure evolution in 2D DEM experiments." GeoCongress 2008, American Society of Civil Engineers, 958-965.

Evans, T.M., and Frost, J.D. (2010). "Multiscale investigation of shear bands in sand: physical and numerical experiments." International Journal for Numerical and Analytical Methods in Geomechanics, 34(15), 1634-1650.

Evans, T.M., Khoubani A., and Montoya, B.M. (2014). "Simulating mechanical response in biocemented sands." Proceedings of the $14^{\text {th }}$ International Conference of the International Association for Compueter Methods and Advances in Geomechanics, 1569-1574, September 22-25, Kyoto, Japan.

Evans, T. M., and Ning, Z. (2013). "Wave propagation in assemblies of cemented spheres." Powders and Grains 2013: Proceedings of the 7th International Conference on Micromechanics of Granular Media. AIP Conference Proceedings, 233-236.

Evans, T.M., and Valdes, J.R. (2011). "The microstructure of particulate mixtures in one-dimensional compression: numerical studies." Granular Matter, 13(5), 657-669.

Fauriel, S., and Laloui, L. (2012). "A bio-chemo-hydro-mechanical model for microbially induced calcite precipitation in soils." Comput.Geotech., 46 104120.

Feng, K., and Montoya, B. (2014). "Behavior of bio-mediated soil in $k_{0}$ loading." New Frontiers in Geotechnical Engineering, Geo-Shanghai 2014, Geotechnical Special Publications 243, ASCE, Reston, VA,1-10.

Feng, K., and Montoya, B. (2015). "Influence of confinement and cementation level on the behavior of microbial-induced calcite precipitated sands under monotonic drained loading." J. Geotech. Geoenviron. Eng., 10.1061/(ASCE)GT.1943-5606.0001379, 04015057.

Hardin, B. O., and Drnevich, V. P. (1972). "Shear modulus and damping in soils." Journal of the Soil Mechanics and Foundations Division, 98(7), 667-692. 
572 Huang, and Airey. (1998). "Properties of artificially cemented carbonate sand." J.Geotech.Geoenviron.Eng., 124(6), 492-499.

574 Ismail, M., Joer, H., Sim, W., and Randolph, M. (2002). "Effect of cement type on 575 shear behavior of cemented calcareous soil." J.Geotech.Geoenviron.Eng., $576 \quad 128(6), 520-529$.

577

Iwashita, K., and Oda, M. (2000). "Micro-deformation mechanism of shear banding process based on modified distinct element method." Powder Technol, 109(1-3), 192-205.

Jacobson, D. E., Valdes, J. R., and Evans, T. M. (2007). "A numerical view into direct shear specimen size effects." Geotechnical Testing Journal, 30(6), 1-5.

Jiang, M., Yu, H., and Leroueil, S. (2007). "A simple and efficient approach to capturing bonding effect in naturally microstructured sands by discrete element method." Int J Numer Methods Eng, 69(6), 1158-1193.

Khoubani, A., Evans, T.M., and Montoya., B.M. (2016). "Particulate simulations of triaxial Tests on bio-cemented sand using a new cementation model." Proceedings of GeoChicago: Sustainability, Energy, and the Geoenvironment, Chicago, IL, August 14-18.

Kuo, C., and Frost, J. (1996). "Uniformity evaluation of cohesionless specimens using digital image analysis." J.Geotech.Engrg., 122(5), 390-396.

Lee, K. L. (1978). "End restraint effects on undrained static triaxial strength of sand." Journal of the Geotechnical Engineering Division, 104(6), 687-704.

Leroueil, S., and Vaughan, P. R. (1990). "The general and congruent effects of structure in natural soils and weak rocks." Géotechnique, 40(3), 467-488.

Lin, H., Suleiman, M.T., Brown, D.G., Kavazanjian, E. (2016). "Mechanical Behavior of Sands Treated by Microbially Induced Carbonate Precipitation." J. Geotech. Geoenviron. Eng.,142(2): 04015066.

Martinez, B., DeJong, J., Ginn, T., Montoya, B., Barkouki, T., Hunt, C., Tanyu, B., and Major, D. (2013). "Experimental optimization of microbial-induced 
600

carbonate precipitation for soil improvement." J.Geotech.Geoenviron.Eng., 139(4), 587-598.

Morad, S. (1998). "Carbonate cementation in sandstones: distribution patterns and geochemical evolution." Carbonate Cementation in Sandstones, Blackwell Publishing Ltd., 1-26.

Montoya, B., and Feng, K. (2015). "Deformation of microbial induced calcite bonded sands: A micro-scale investigation." Proceedings from the Sixth International Symposium on Deformation Characteristics of Geomaterials, Buenos Aires,978-985.

Montoya, B.M., and DeJong, J.T. (2015). "Stress-Strain behavior of sands cemented by microbially induced calcite precipitation." J. Geotech. Geoenviron. Eng., 10.1061/(ASCE)GT.1943-5606.0001302, 04015019.

Mortensen, B. M., Haber, M. J., DeJong, J. T., Caslake, L. F., and Nelson, D. C. (2011). "Effects of environmental factors on microbial induced calcium carbonate precipitation." J.Appl.Microbiol., 111(2), 338-349.

Narsilio, G.A., and Santamarina, J.C. (2008). "Terminal densities." Géotechnique, 58(8), 669-674.

$\mathrm{Ng}, \mathrm{T}$. , and Wang, C. (2001). "Comparison of a 3-D DEM simulation with MRI data." Int.J.Numer.Anal.Methods Geomech., 25(5), 497-507.

Ni, Q., Powrie, W., Zhang, X., and Harkness, R. (2000). "Effect of particle properties on soil behavior: 3-D Numerical Modeling of Shearbox Tests." GeoDenver 2000, American Society of Civil Engineers, Denver, 58-70.

Potyondy, D.O., and Cundall, P.A. (2004). "A bonded-particle model for rock." International Journal of Rock Mechanics \& Mining Sciences, 41, 1329-1364.

Qabany, A. A., and Soga, K. (2013). "Effect of chemical treatment used in MICP on engineering properties of cemented soils." Géotechnique, 63(4), 331-339.

Santamarina, J. C., Klein, A., and Fam, M. A. (2001). Soils and waves. John Wiley \& Sons, Chichester, UK. 
Saxena, S. K., Avramidis, A. S., and Reddy, K. R. (1988). "Dynamic moduli and damping ratios for cemented sands at low strains." Can.Geotech.J., 25(2), 353368.

Shanahan, C., and Montoya, B. (2014). "Strengthening coastal sand dunes using microbial-induced calcite precipitation." GeoCongress 2014, American Society of Civil Engineers, 1683-1692.

Stokoe, K. H. I., Lee, S. H. H., and Knox, D. P. (1985). "Shear moduli measurements under true triaxial stresses." Proc., Adv. in the Art of Testing Soil Under Cyclic Conditions, ASCE, New York, 166-185.

Struble, L., and Sun, G. (1995). "Viscosity of Portland cement paste as a function of concentration." Adv.Cem.Based Mater., 2(2), 62-69.

Suiker, A. S. J., and Fleck, N. A. (2004). "Frictional collapse of granular assemblies." Journal of Applied Mechanics, Transactions ASME, 71(3), 350-358.

Tagliaferri, F., Waller, J., Ando, E., Hall, S., Viggiani, G., Besuelle, P., and DeJong, J. (2011). "Observing strain localisation processes in bio-cemented sand using $\mathrm{X}$ ray imaging." Granular Matter, 13(3), 247-250.

Thornton, C. (2000). "Numerical simulations of deviatoric shear deformation of granular media." Géotechnique, 50(1), 43-53.

van Paassen, L., Ghose, R., van, d. L., van, d. S., and van Loosdrecht, M. (2010). "Quantifying biomediated ground improvement by ureolysis: Large-Scale biogrout experiment." J.Geotech.Geoenviron.Eng., 136(12), 1721-1728.

Wang, L., Frost, J., and Lai, J. (2004). "Three-Dimensional digital representation of granular material microstructure from X-Ray tomography imaging." J.Comput.Civ.Eng., 18(1), 28-35.

Wang, Y., and Leung, S. (2008). "Characterization of cemented sand by experimental and numerical investigations." J.Geotech.Geoenviron.Eng., 134(7), 992-1004. 
655 Whiffin, V. S., Van Paassen, L. A., and Harkes, M. P. (2007). "Microbial carbonate 656 precipitation as a soil improvement technique." Geomicrobiology Journal, 657 24(5), 417-423.

658 Zhang, L., and Thornton, C. (2007). "A numerical examination of the direct shear 659 test." Géotechnique, 57(4), 343-354.

660 Zhao, X., and Evans, T. (2009). "Discrete simulations of laboratory loading 661 conditions." Int.J.Geomech., 9(4), 169-178.

662

663 
Table 1. Sand Characteristics

\begin{tabular}{ccccccc}
\hline$D_{\text {50 }}(\mathrm{mm})$ & $C_{u}$ & $C_{c}$ & $G_{S}$ & $e_{\min }$ & $e_{\max }$ & Shape \\
\hline 0.22 & 1.4 & 0.9 & 2.65 & 0.55 & 0.87 & Round \\
\hline
\end{tabular}

665

666

667

Table 2. Chemical Recipe of Injection Media

\begin{tabular}{ccc}
\hline Chemical Name & $\begin{array}{c}\text { Biological Injection } \\
\text { Concentrations (mM) }\end{array}$ & $\begin{array}{c}\text { Cementation Injection } \\
\text { Concentrations (mM) }\end{array}$ \\
\hline Urea & 333 & 333 \\
Ammonium Chloride & 374 & 374 \\
Calcium Chloride & 0 & 50 \\
\hline
\end{tabular}

668

669

670

671

672

673

674

675

676

677

678

679

680 
Parameters

\section{Particle Properties}

Particle normal stiffness

Particle shear stiffness

Particle friction coefficient

Particle specific gravity

Particle aspect ratio

Number of particles

Wall Properties

Platen stiffness

Membrane stiffness

Platen friction coefficient

\section{Bond Properties}

Bond radius for light MICP cementation Bond radius for moderate MICP cementation

Bond radius for heavy MICP cementation

Normal contact stiffness

Shear contact stiffness

Normal bond strength

Shear bond strength

\section{Selected Values}

$$
\begin{gathered}
2 \times 10^{8} \mathrm{~N} / \mathrm{m} \\
5 \times 10^{6} \mathrm{~N} / \mathrm{m} \\
0.5 \\
2.65 \\
1.5 \\
\sim 14,000
\end{gathered}
$$

$2 \times 10^{8} \mathrm{~N} / \mathrm{m}$

1.0
0.25

0.3

0.5

$5 \times 10^{8} \mathrm{~Pa} / \mathrm{m}$

$1 \times 10^{9} \mathrm{~Pa} / \mathrm{m}$

$5 \times 10^{6} \mathrm{~Pa}$

$1 \times 10^{6} \mathrm{~Pa}$ 
Figure 1. (a) Stress-strain behavior and (b) volumetric deformation of untreated numerical and experimental specimens with varying relative density under $100 \mathrm{kPa}$ confinement, for each

690 results presented, initial density is indicated. Solid lines represent numerical results and data 691 points represent experimental results.

Figure 2. (a) Stress-strain behavior and (b) volumetric deformation of treated numerical and 694 experimental specimens with varying cementation levels under $100 \mathrm{kPa}$ confinement, for each 695 triaxial results presented, cementation level is indicated. Solid lines represent numerical result 696 and data points represent experimental results.

Figure 3. Grain size distribution of experimental and numerical soil particles.

701 (SEM) image of soil particle and cementation at 500x magnificent and (c) SEM image at 1000x 702 magnification.

704 Figure 5. Comparison of (a) initial stiffness (b) peak friction angle and (c) residual friction angle 705 between numerical and experimental specimens, for each results presented, solid symbols 
represent uncemented specimens and half solid symbols represent cemented specimens; initial

707 density (for untreated specimen) or cementation level (for cemented specimen) is indicated

708 (e.g., solid symbols with label loose represents the loose untreated specimen; half solid symbols

709 with label light represents the lightly cemented specimen).

711 Figure 6. Void ratio evolution of untreated and treated numerical specimens during shearing,

712 for each results presented, solid symbols represent uncemented specimens and hollow symbols

713 represent cemented specimens; initial density (for untreated specimen) or cementation level

714 (for cemented specimen) is indicated (e.g., solid symbols with label loose represents the loose

715 untreated specimen; hollow symbols with label light represents the lightly cemented specimen).

717 Figure 7. Meso scale void ratio of untreated and treated numerical specimens during shearing.

718 (a) uncemented loose specimen under $100 \mathrm{kPa}$ confinement (b) uncemented medium dense

719 specimen under $100 \mathrm{kPa}$ confinement (c) uncemented dense specimen under $100 \mathrm{kPa}$

720 confinement (d) lightly cemented specimen under $100 \mathrm{kPa}$ confinement (e) moderately

721 cemented specimen under $100 \mathrm{kPa}$ confinement (f) heavily cemented specimen under $100 \mathrm{kPa}$

722 confinement $(\mathrm{g})$ heavily cemented specimen under $400 \mathrm{kPa}$ confinement.

724 Figure 8. Coordination number evolution of untreated and treated numerical specimens during 725 shearing, for each results presented, solid symbols represent uncemented specimens and 
726 hollow symbols represent cemented specimens; initial density (for untreated specimen) or 727 cementation level (for cemented specimen) is indicated (e.g., solid symbols with label loose 728 represents the loose untreated specimen; hollow symbols with label light represents the lightly 729 cemented specimen).

730

731 Figure 9. Bond breakage percentage of treated numerical specimens and estimated bond 732 breakage percentage of experimental specimens, for each results presented, solid symbols 733 represent numerical specimens and half solid and hollow symbols represent physical specimens; 734 cementation level is indicated (e.g., light represents a lightly cemented simulated specimen).

736 Figure 10. Relationship between shear wave velocity and estimated bond breakage of the 737 moderately cemented physical specimen that is illustrated in Figure 9.

739 Figure 11. Bond breakage percentage of heavily treated specimens under varying confinements, 740 for each result presented, the associated confining pressure is indicated.

742 Figure 12. Position projection of intact bonds (red color) and broken bonds (blue color) at 743 vertical plane under $1 \%(\mathrm{a})$ and $10 \%(\mathrm{~b})$ axial strain. 
745 Figure 13. Stress-strain and bond breakage percentage of heavily cemented numerical 746 specimen during shearing.

747

748 Figure 14. Bond breakage percentage and coordination number of heavily cemented numerical 749 specimen during shearing.

750

751 Figure 15. Stress-strain and dilatancy of the experimental and numerical uncemented loose 752 specimen under shearing.

753

754 Figure 16. Stress-strain and dilatancy of the experimental and numerical uncemented dense 755 specimen under shearing.

756

757 Figure 17. Stress-strain and dilatancy of (a) experimental and (b) numerical heavily cemented 758 specimen under shearing. 
a)

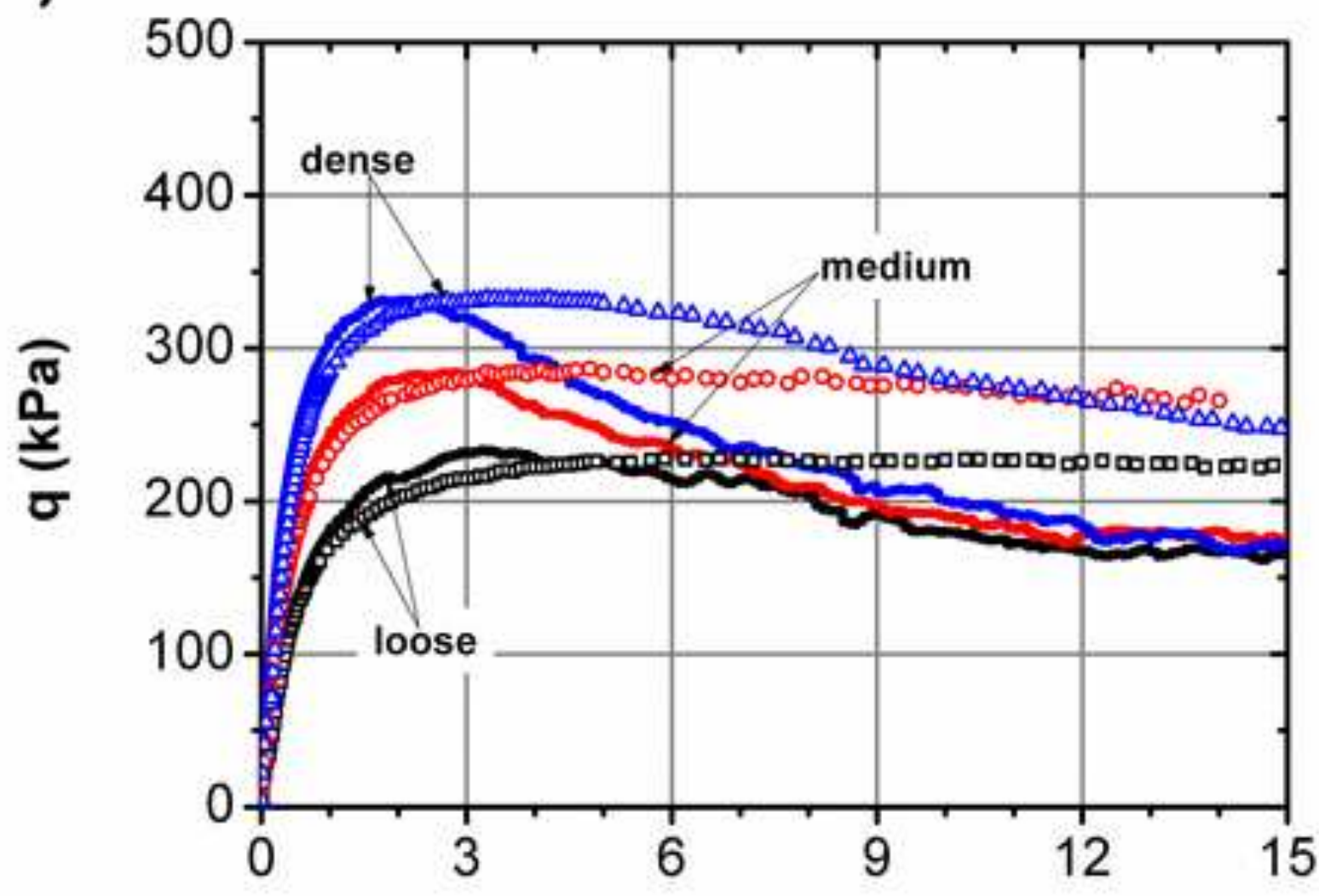

Axial Strain (\%)

b)

¿

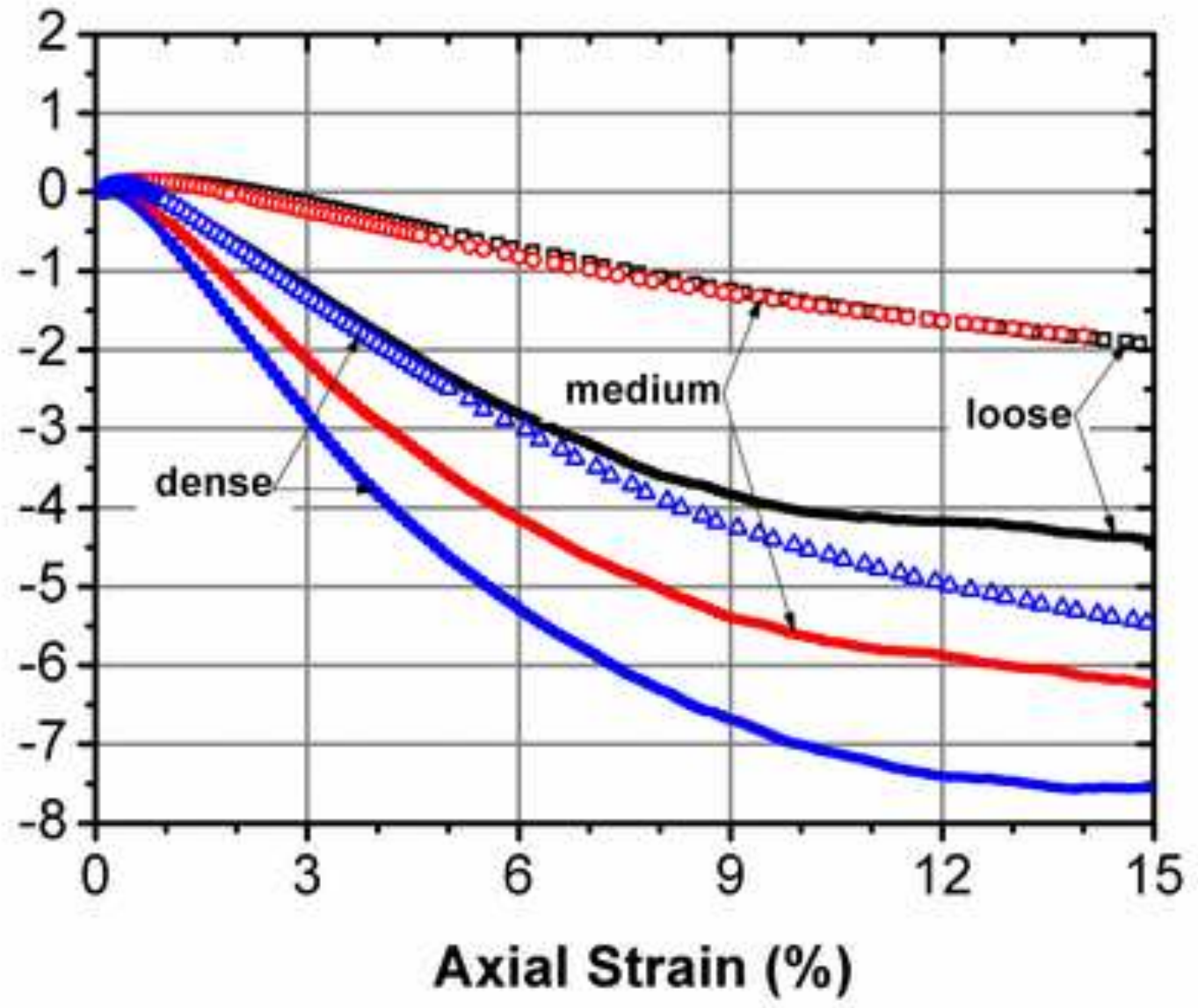


Figure 2

a)

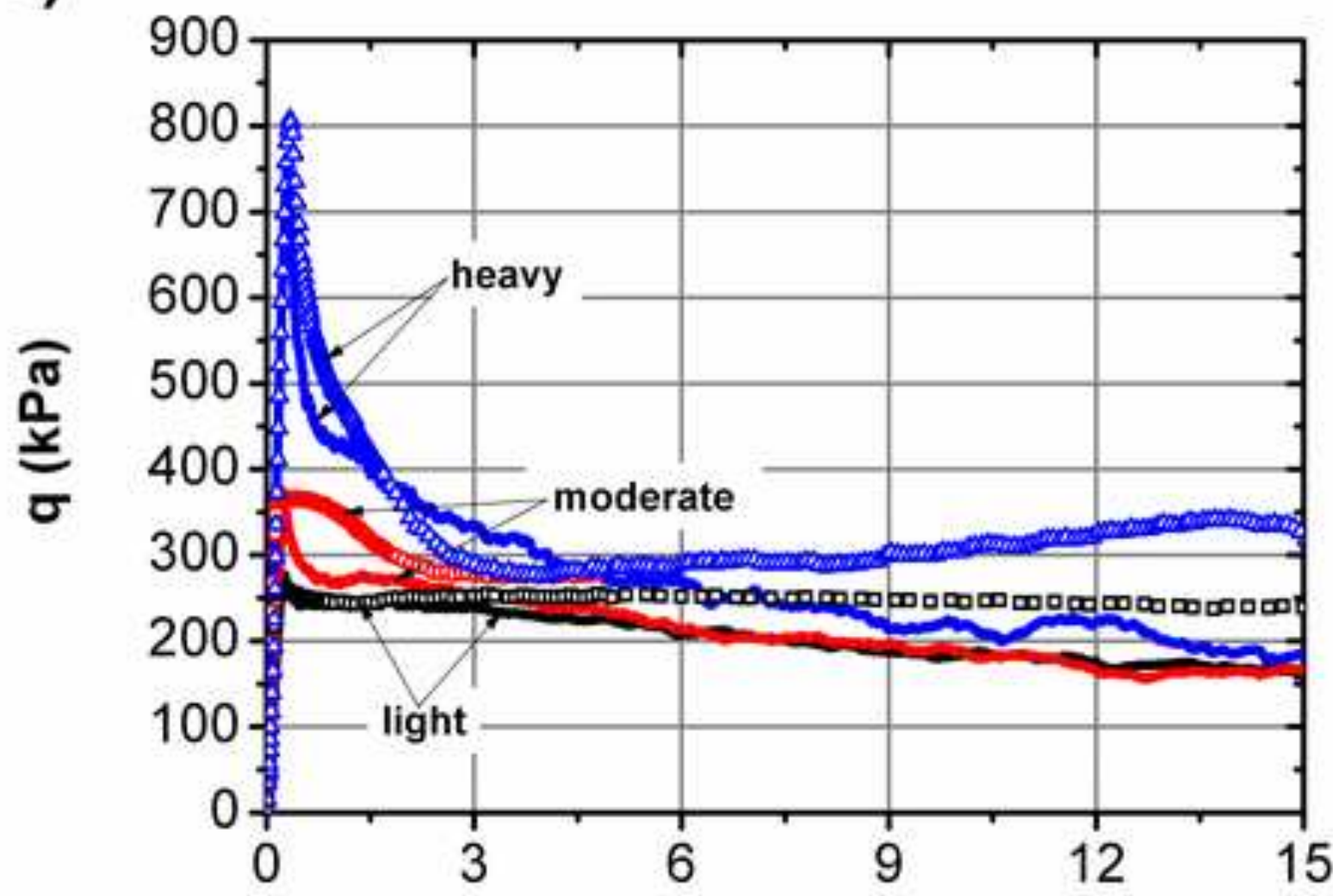

b)

Axial Strain (\%)

ڤั)

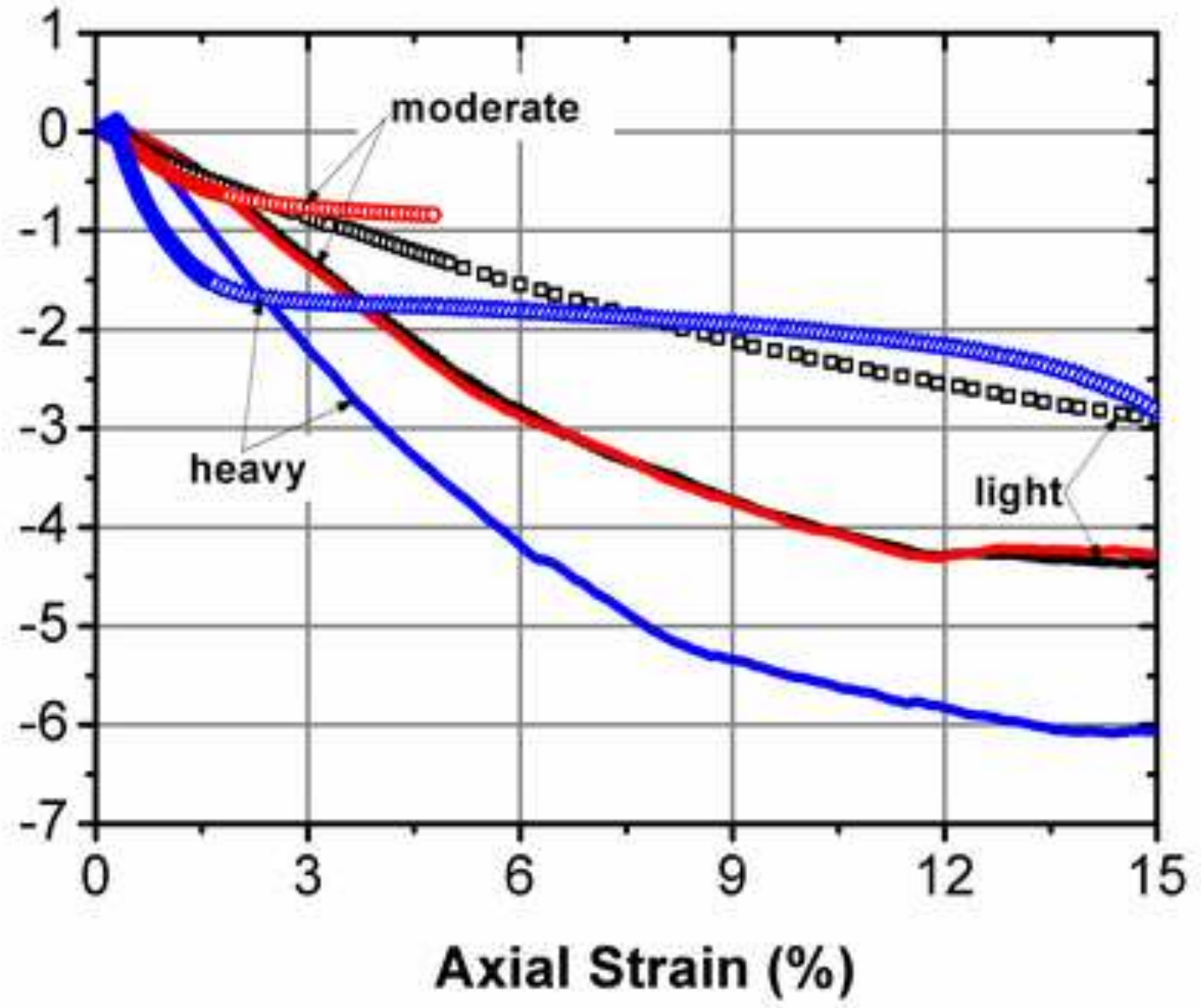




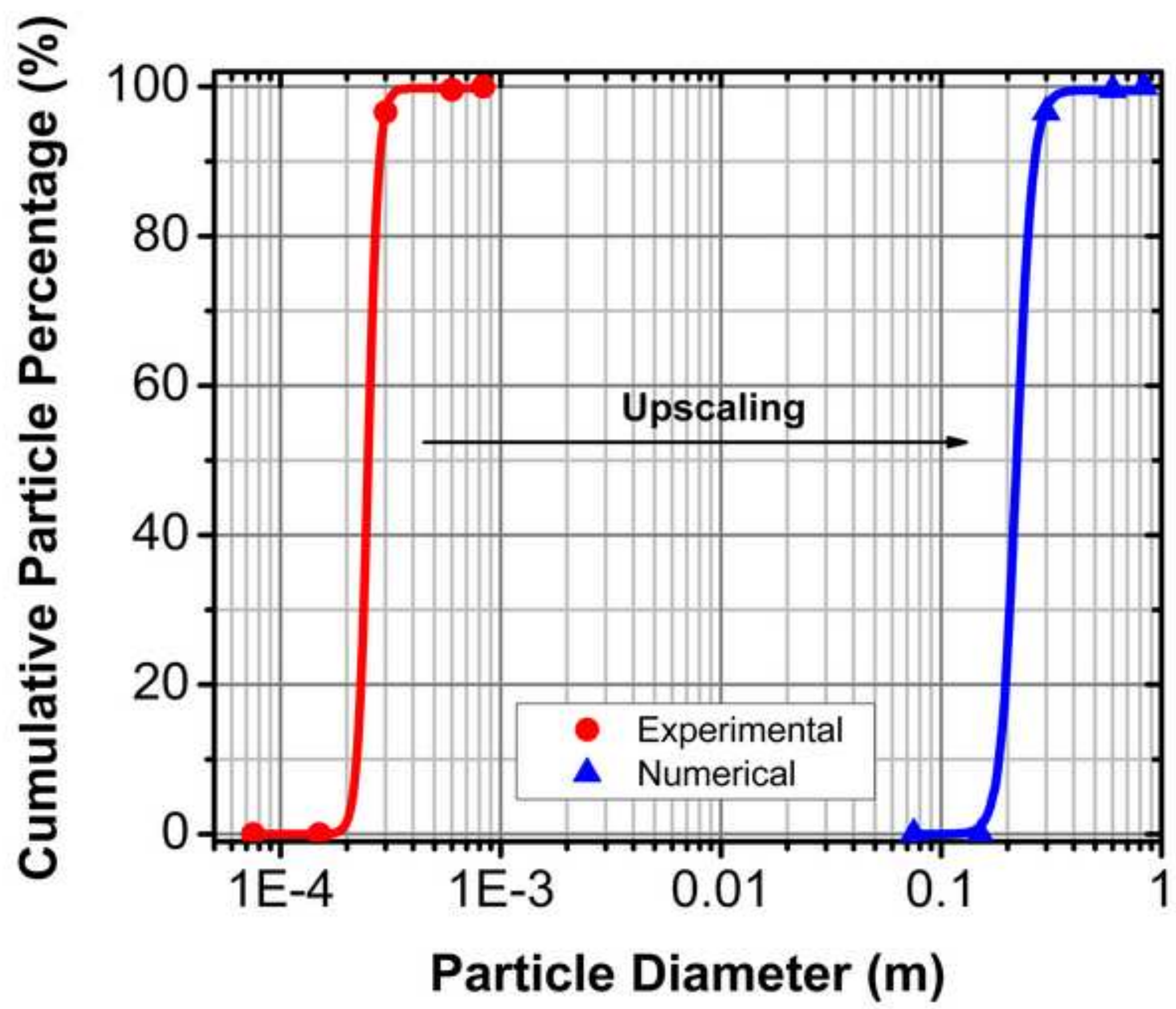


a)

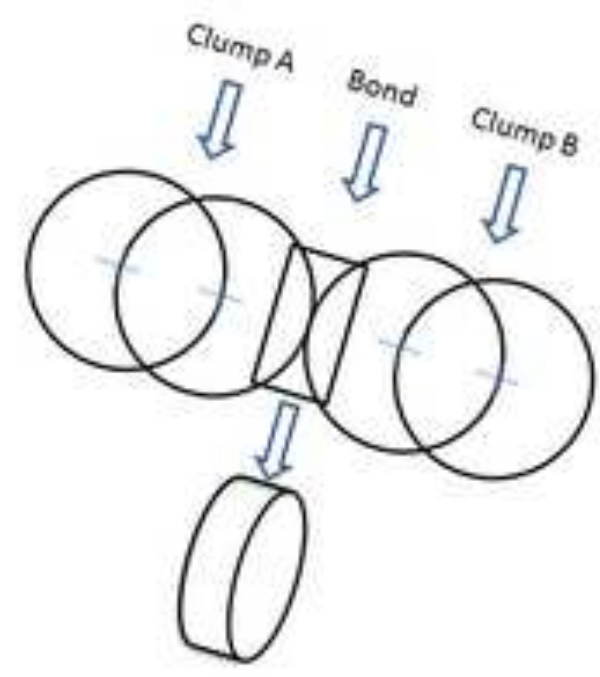

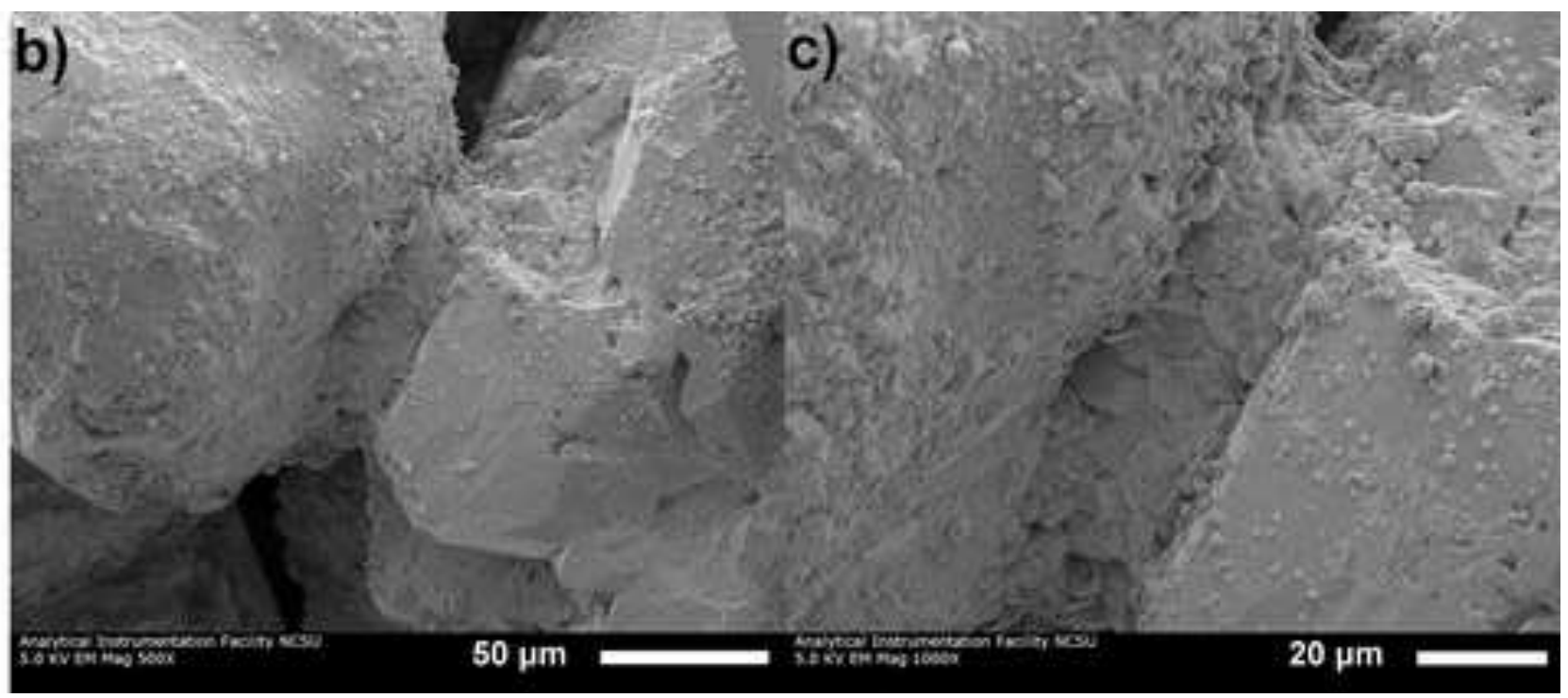


a)

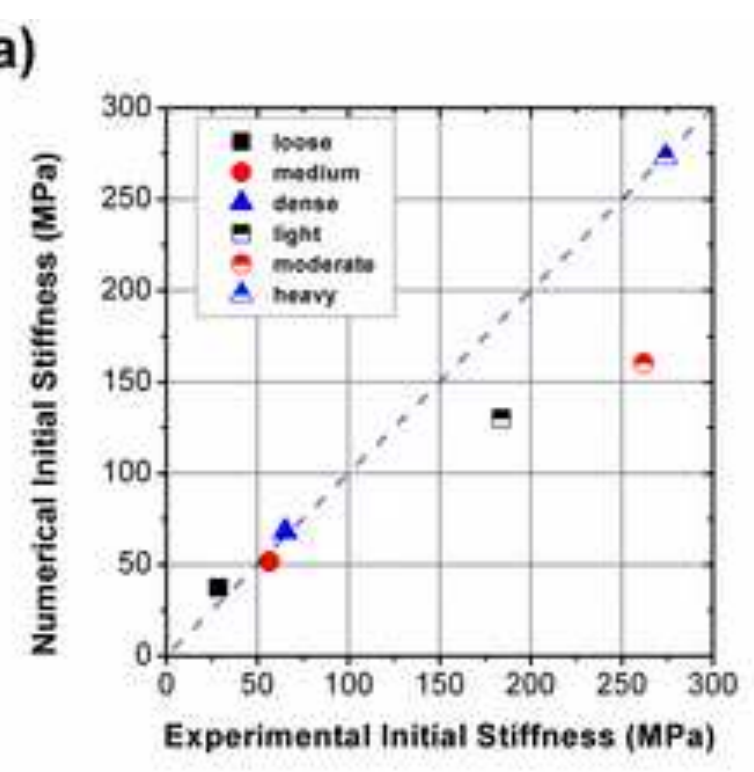

b)

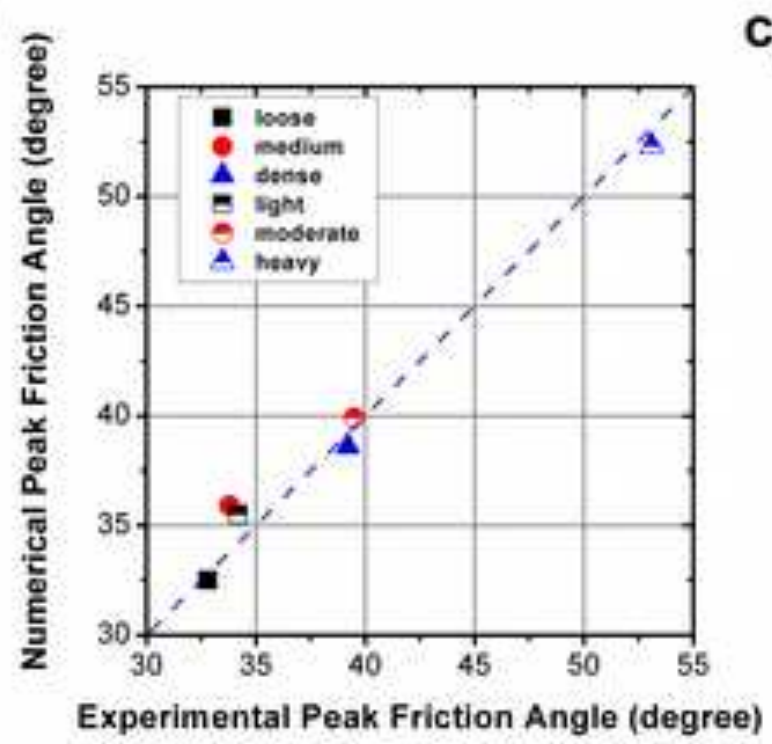

c) $\bar{\Phi}$

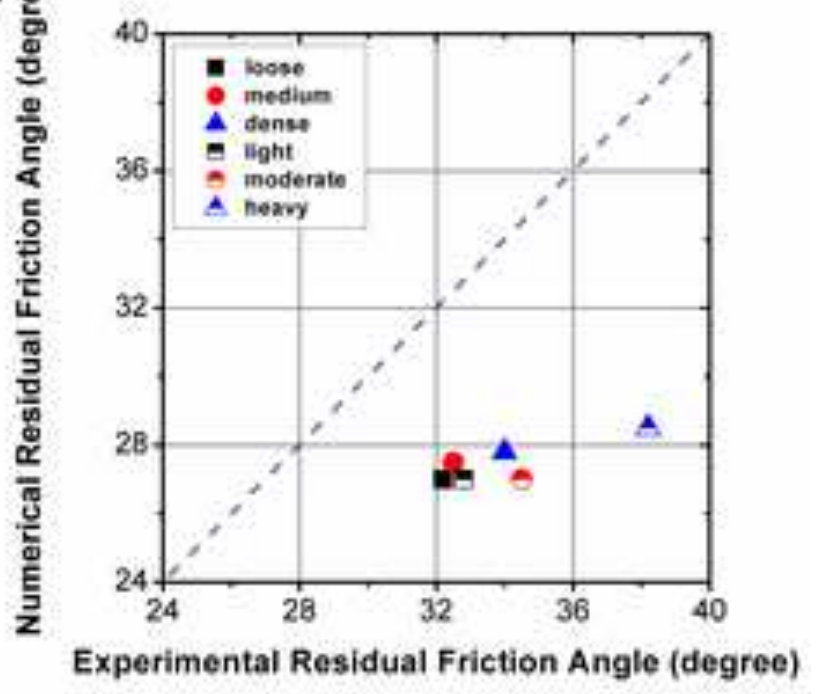




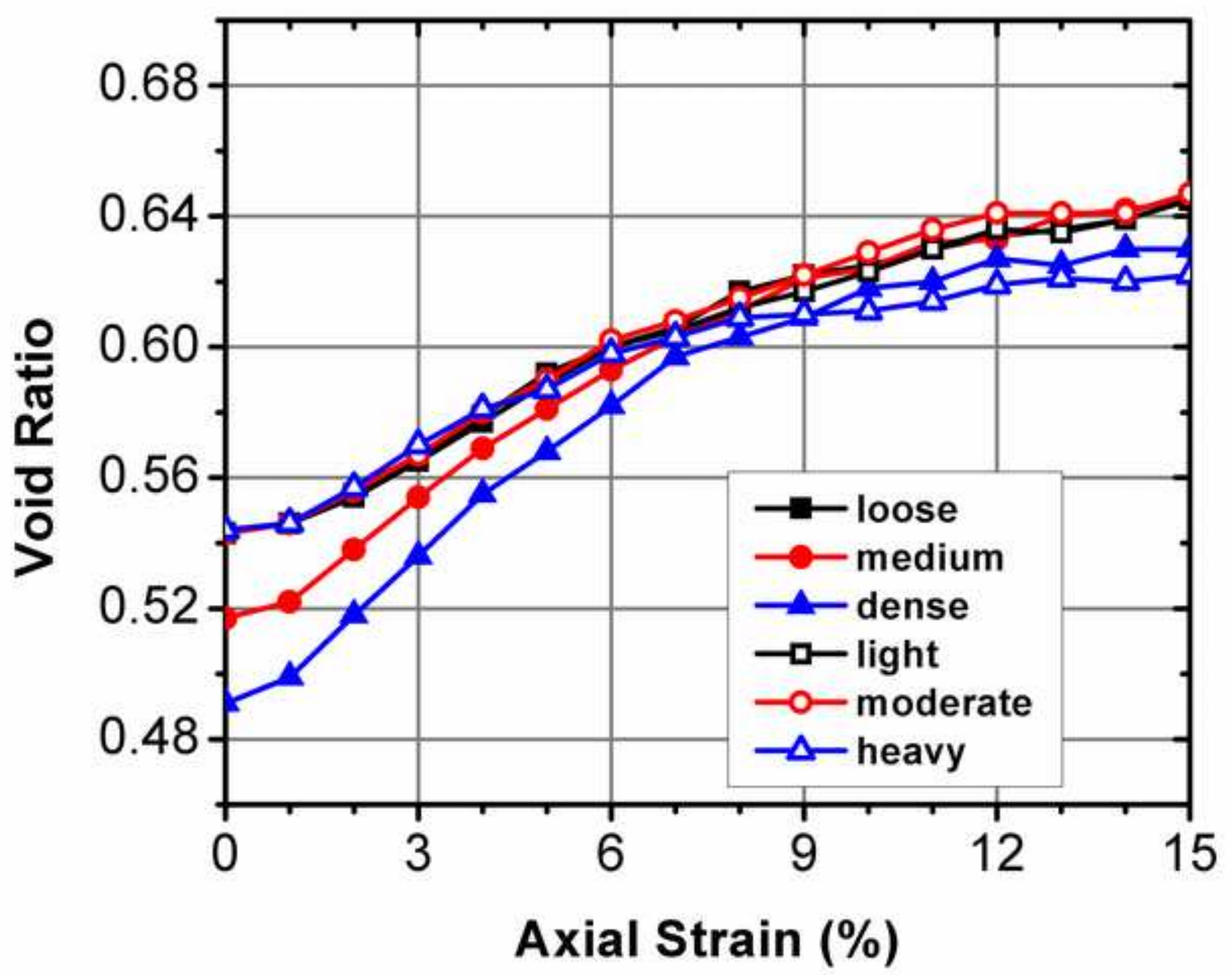



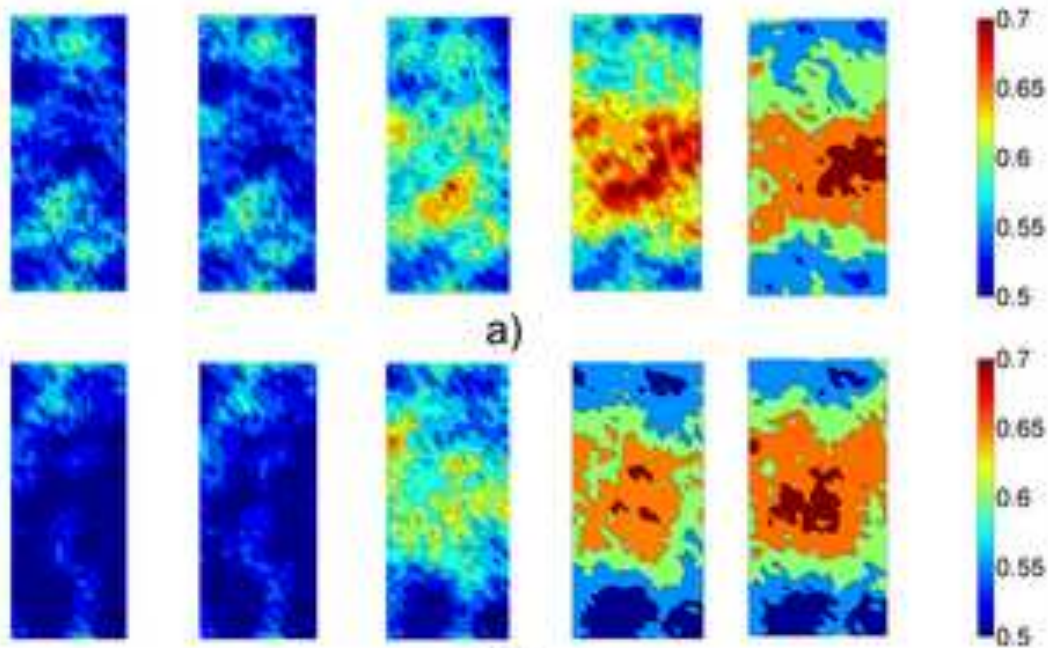

a)
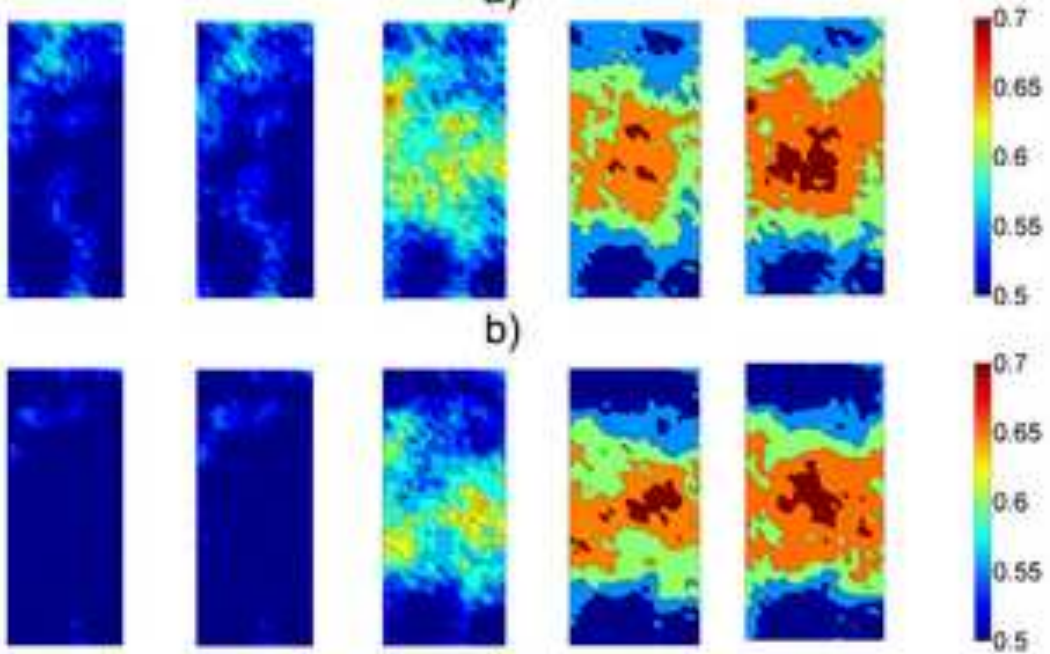

c)
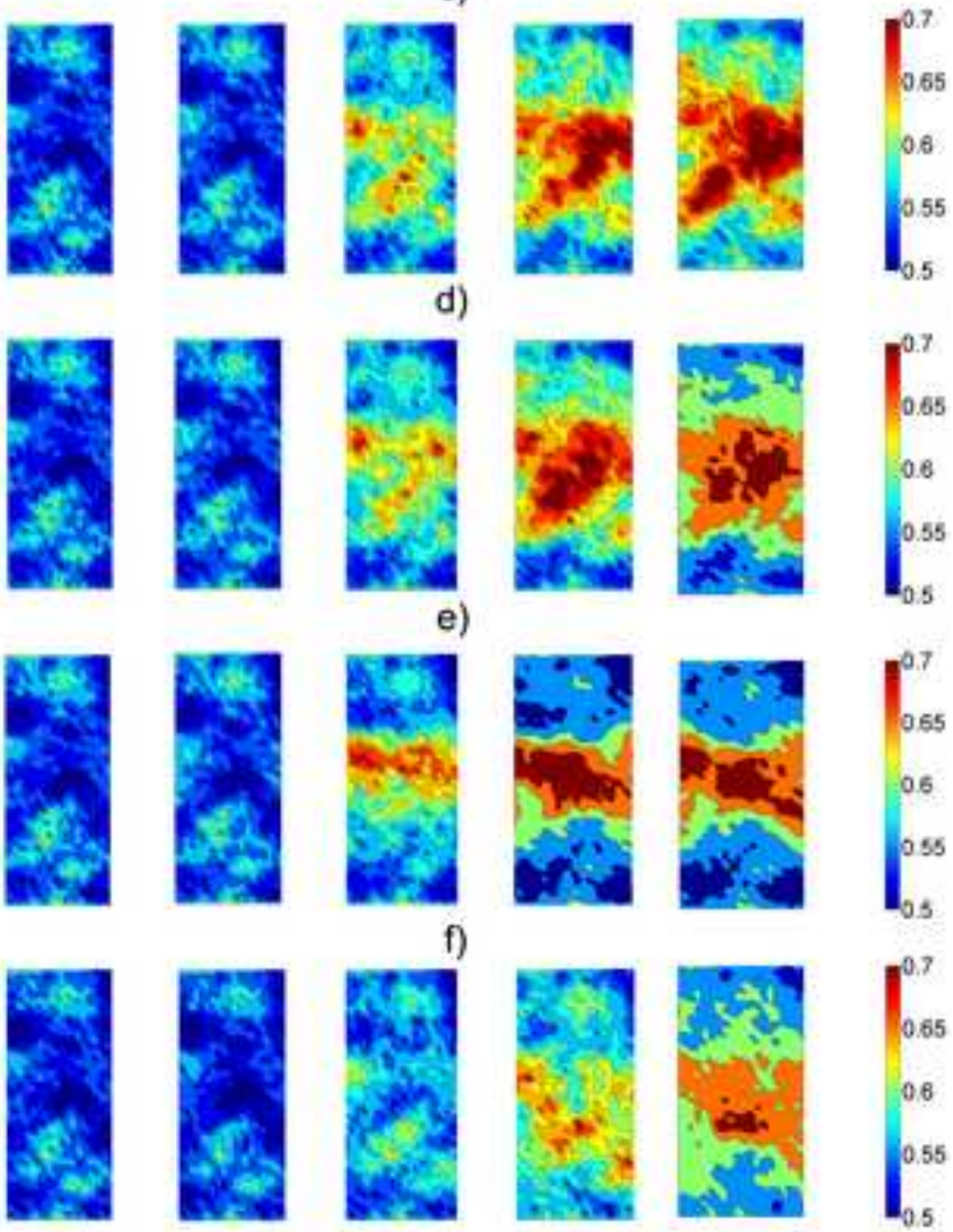

g) 


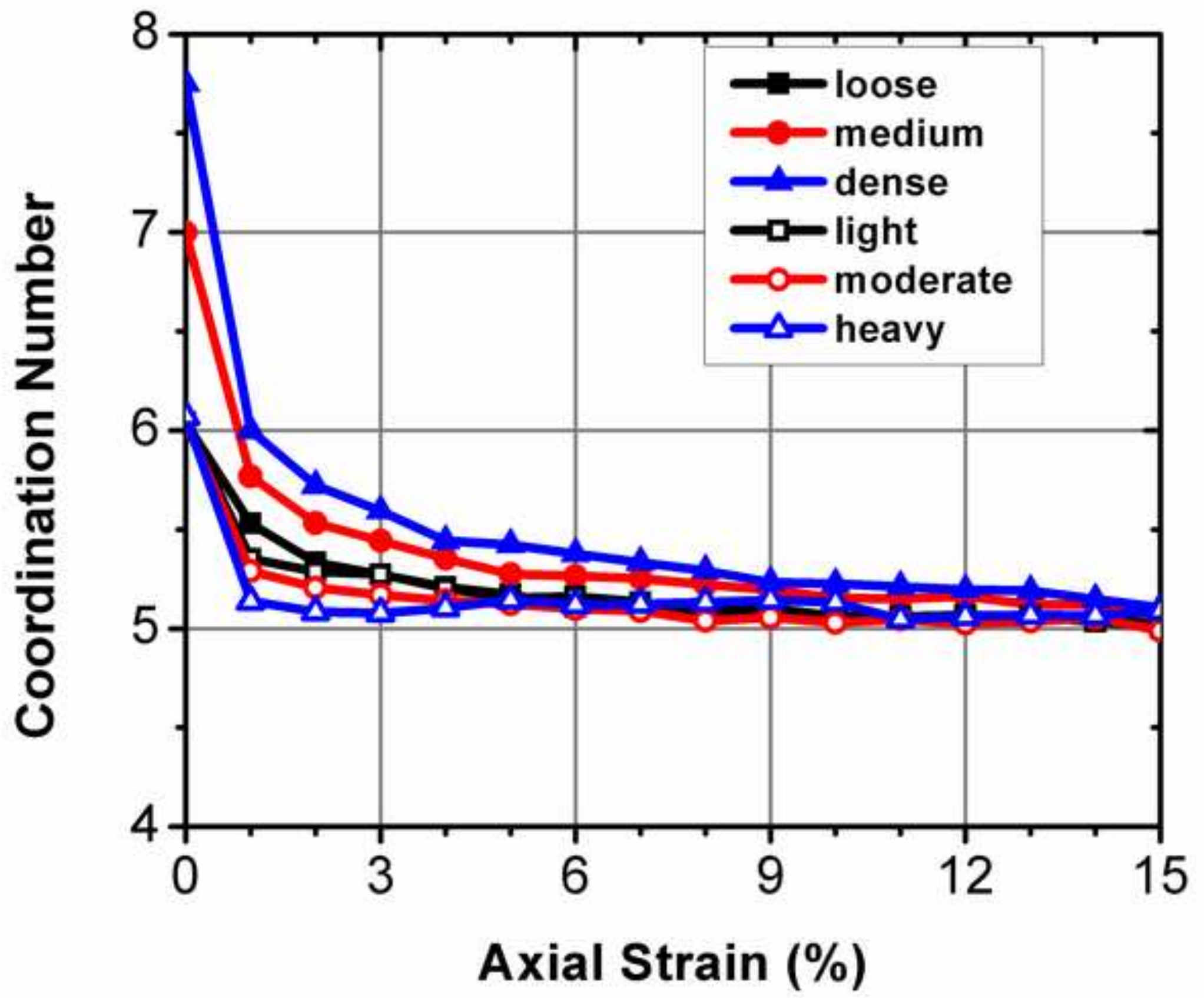




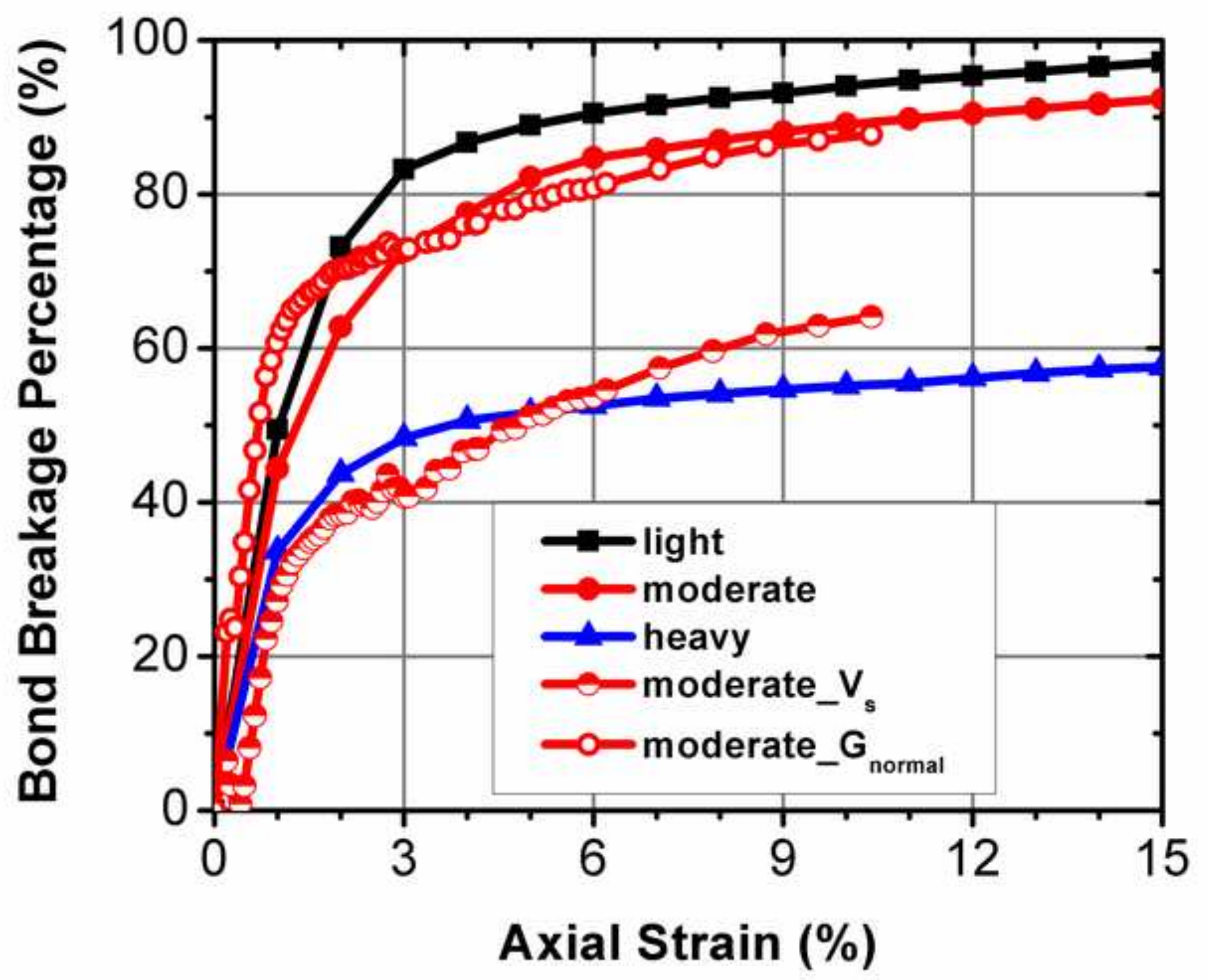



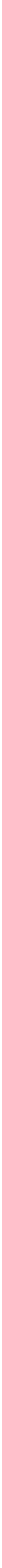


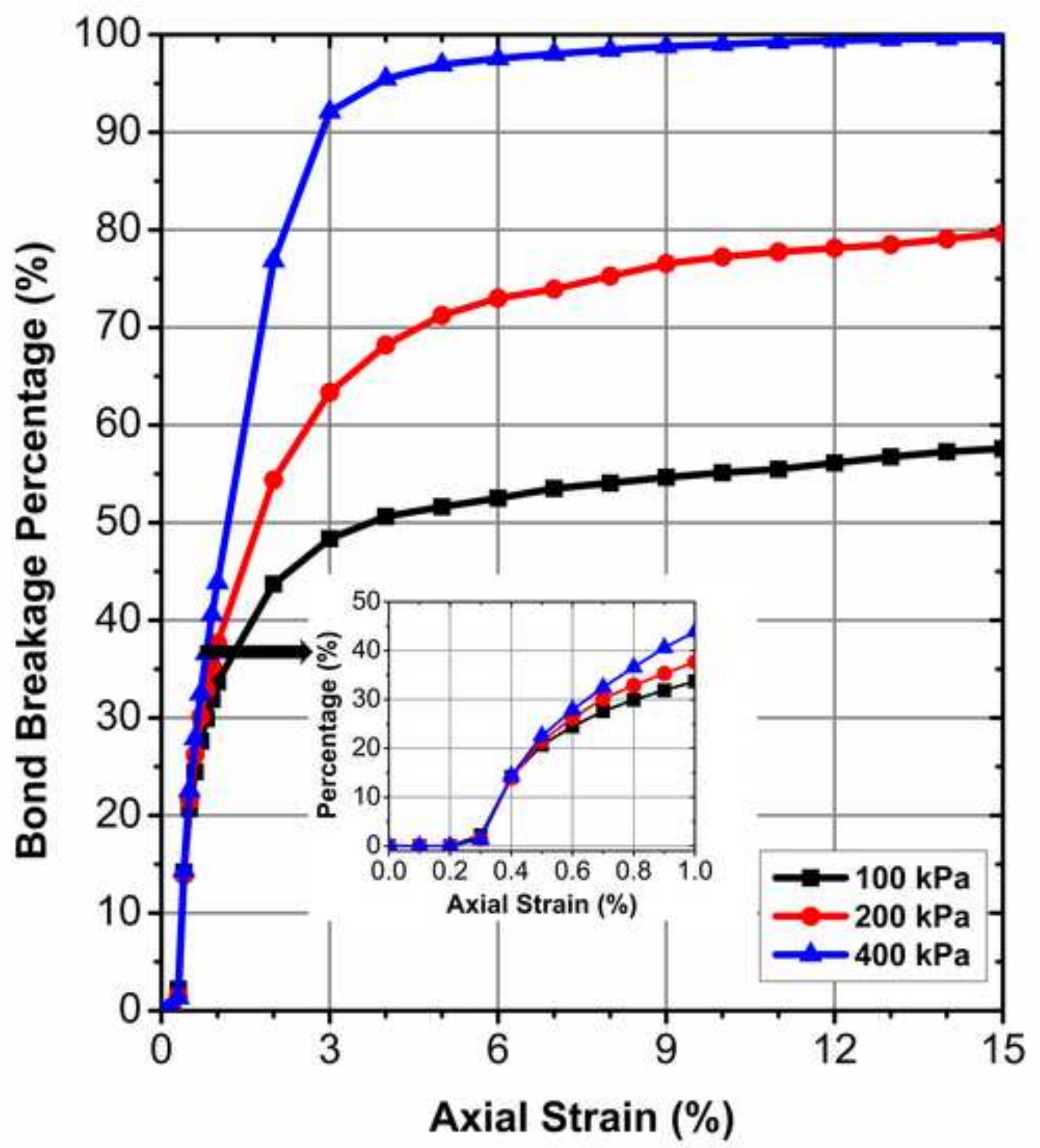

Figure 11 


\section{a)}

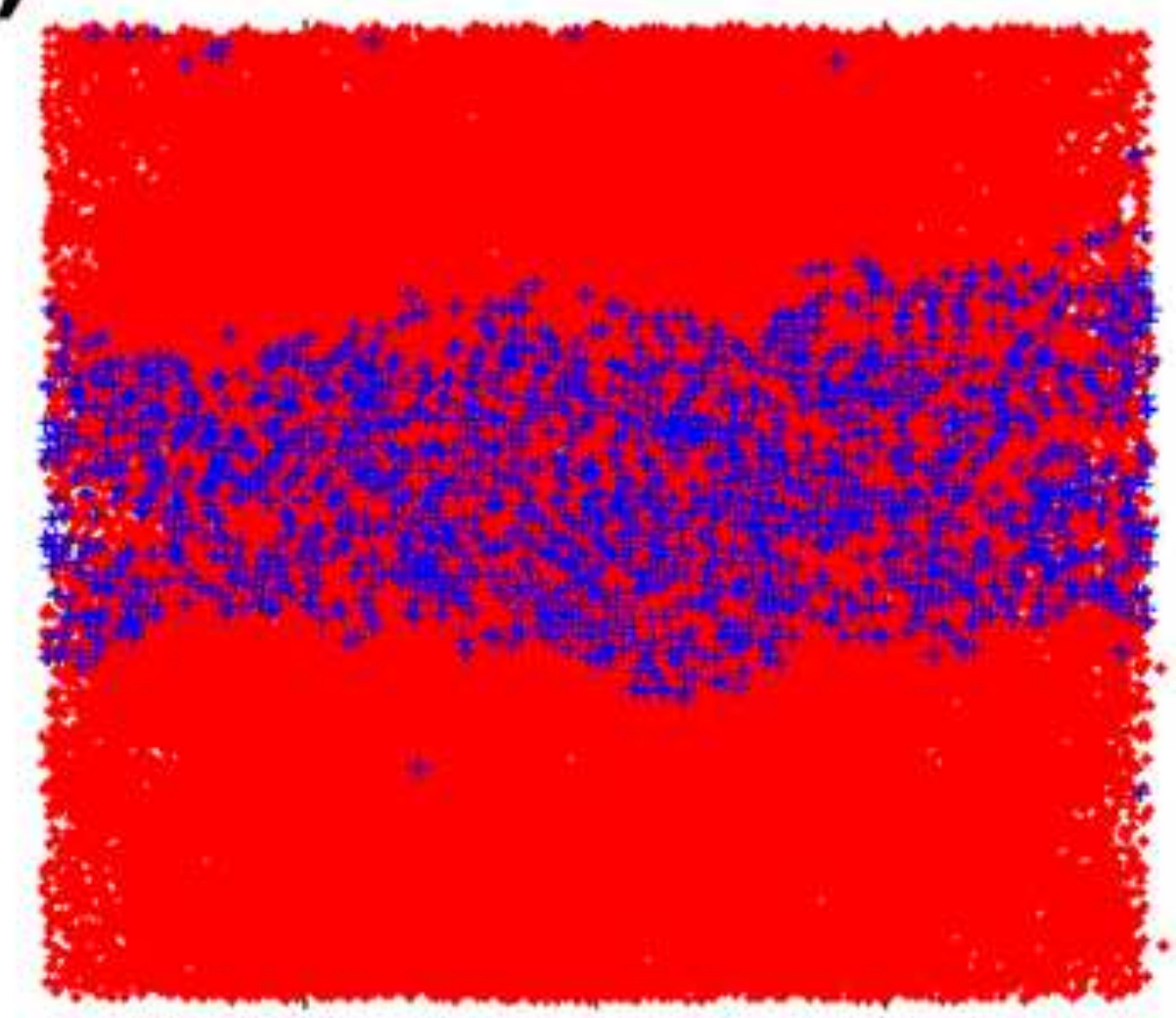

b)

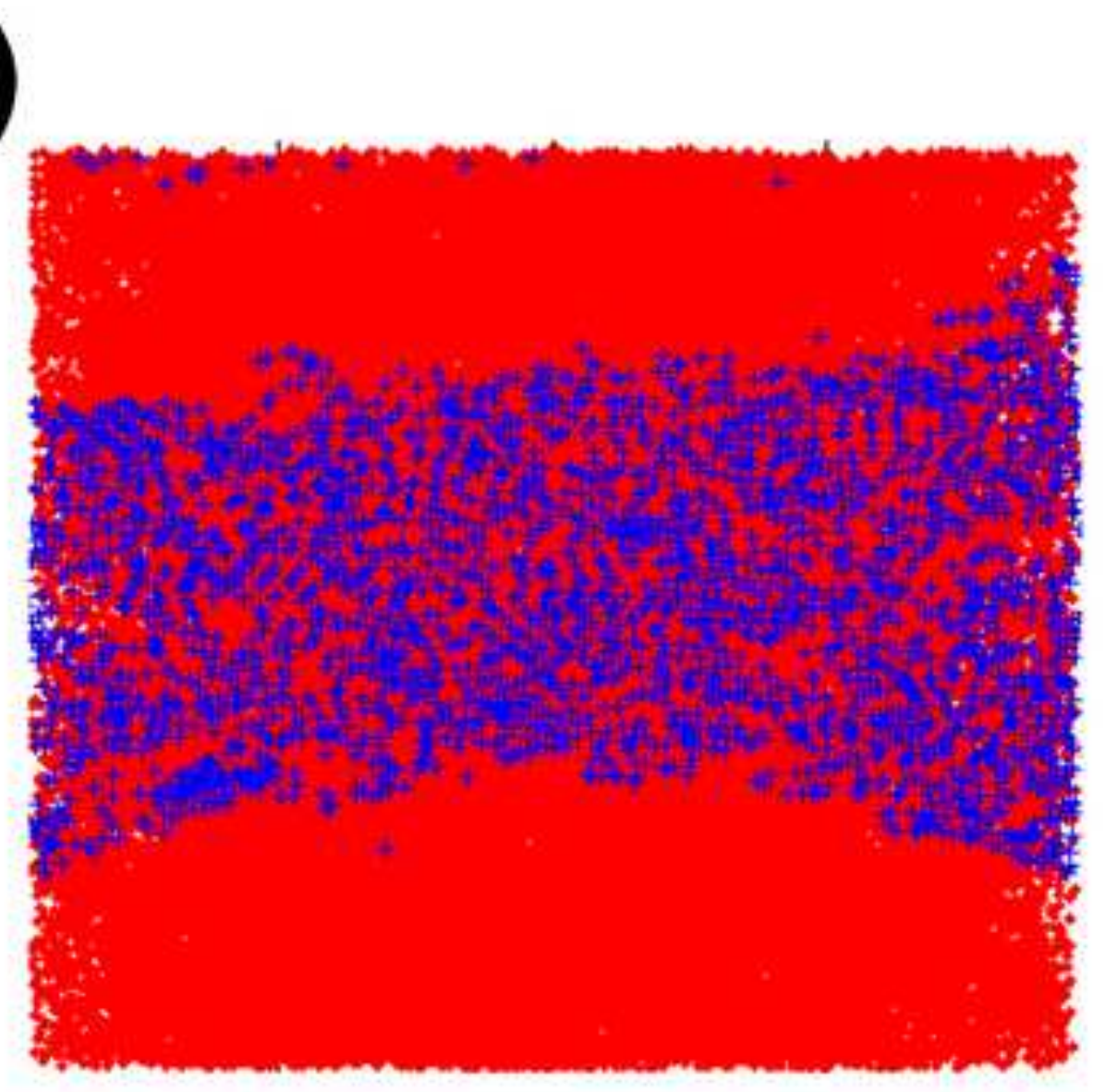

Figure 12 政

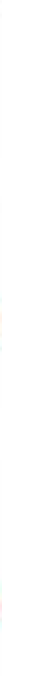

)

Figure 12 


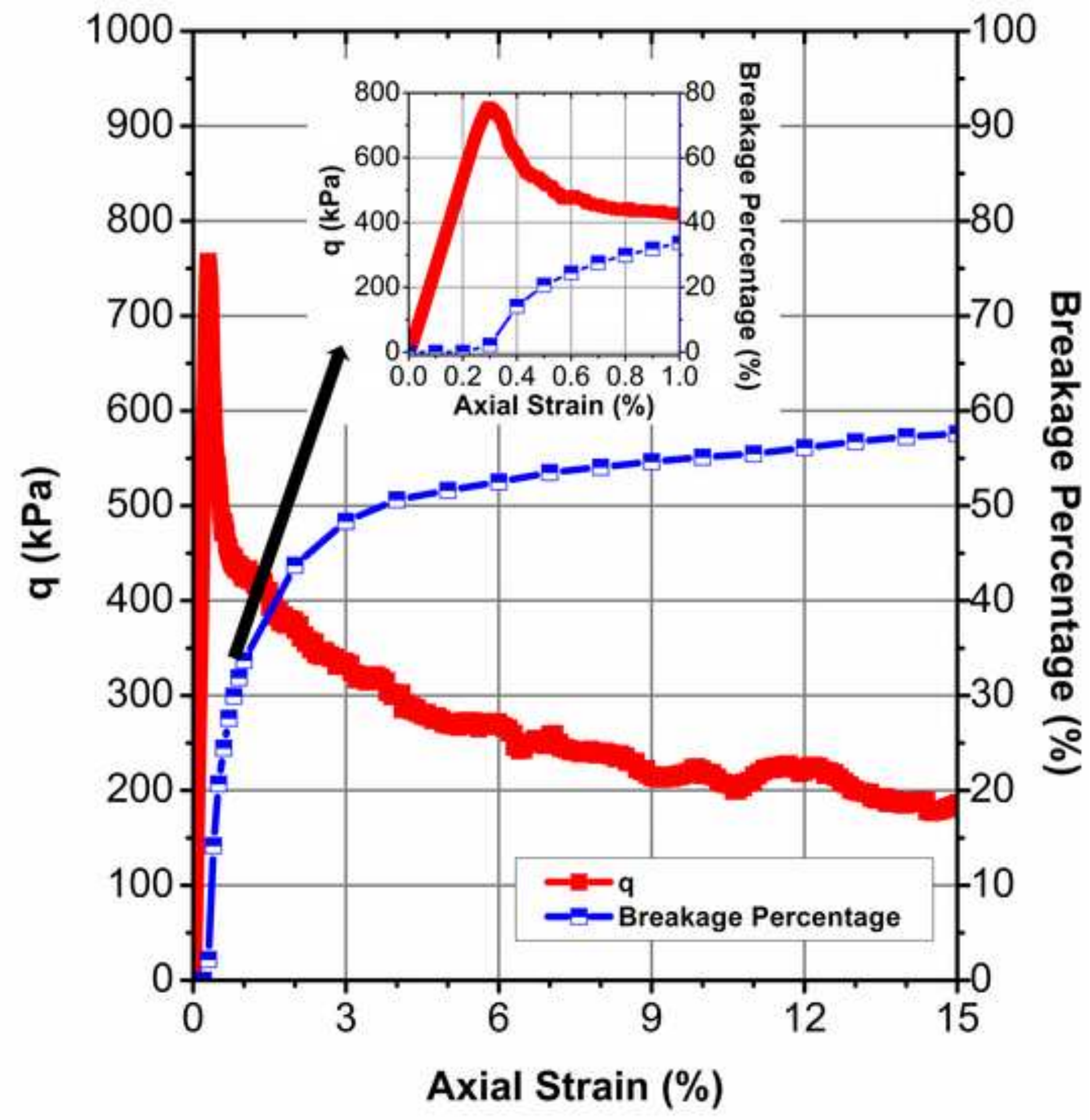

Figure 13 


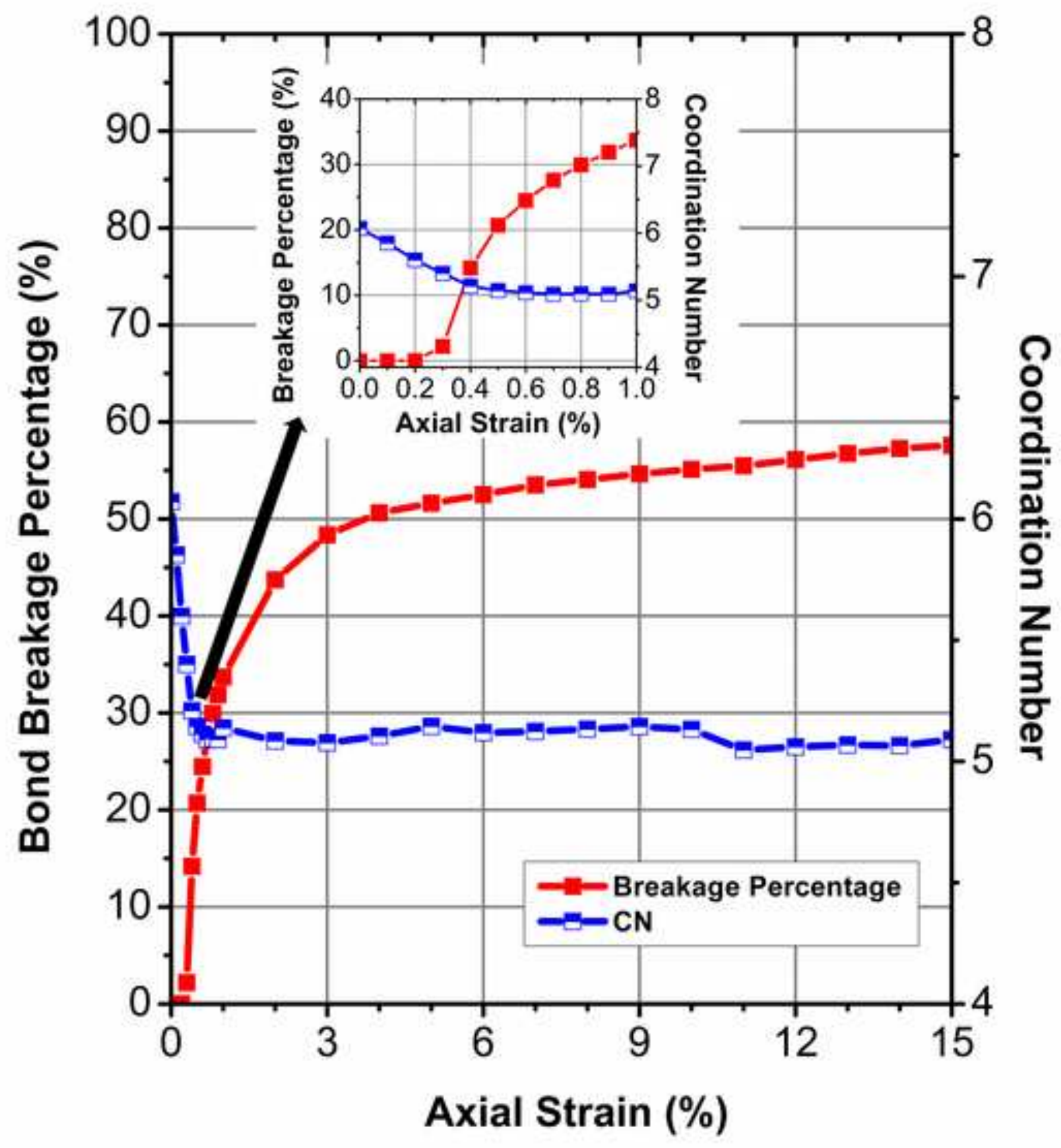

Figure 14 


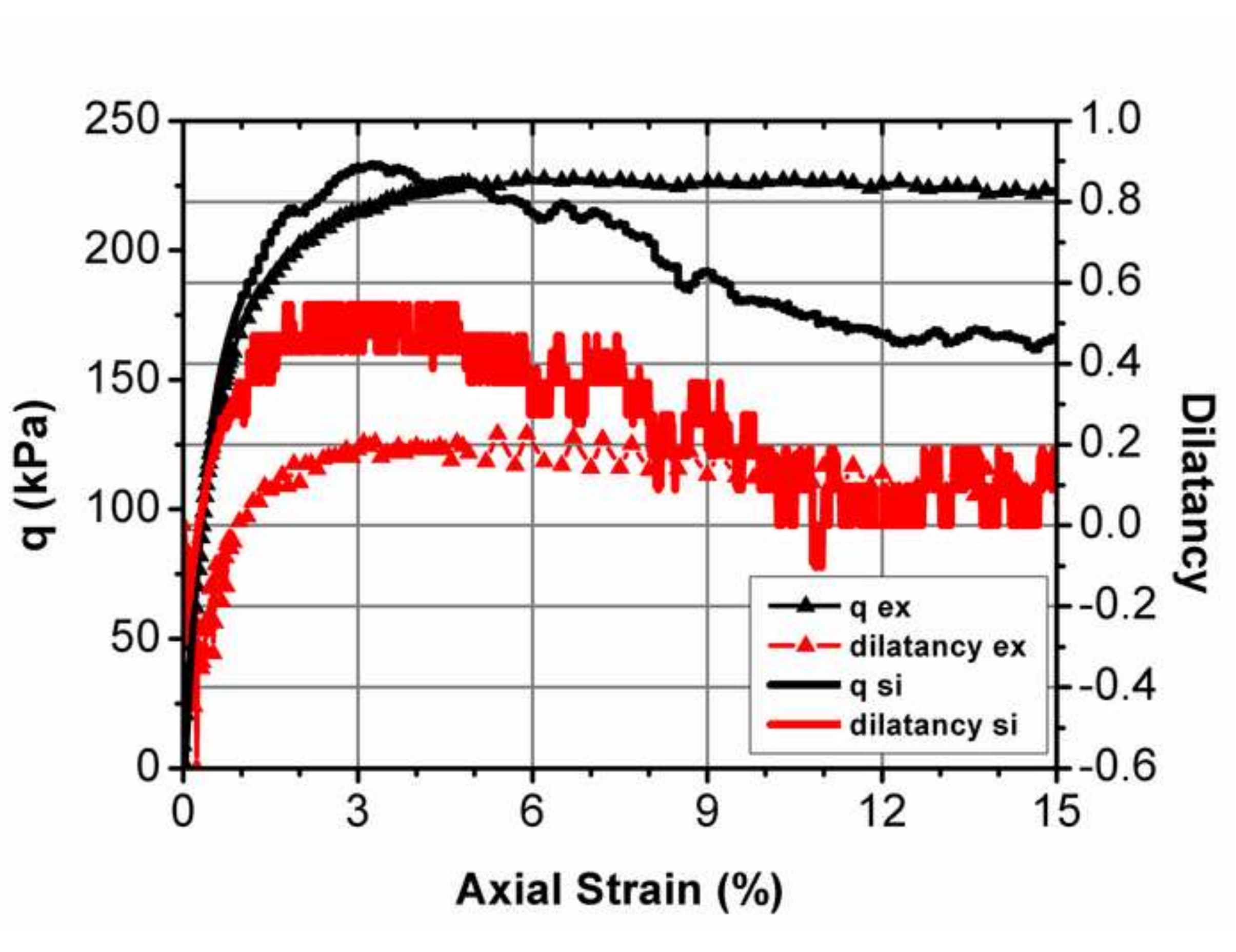




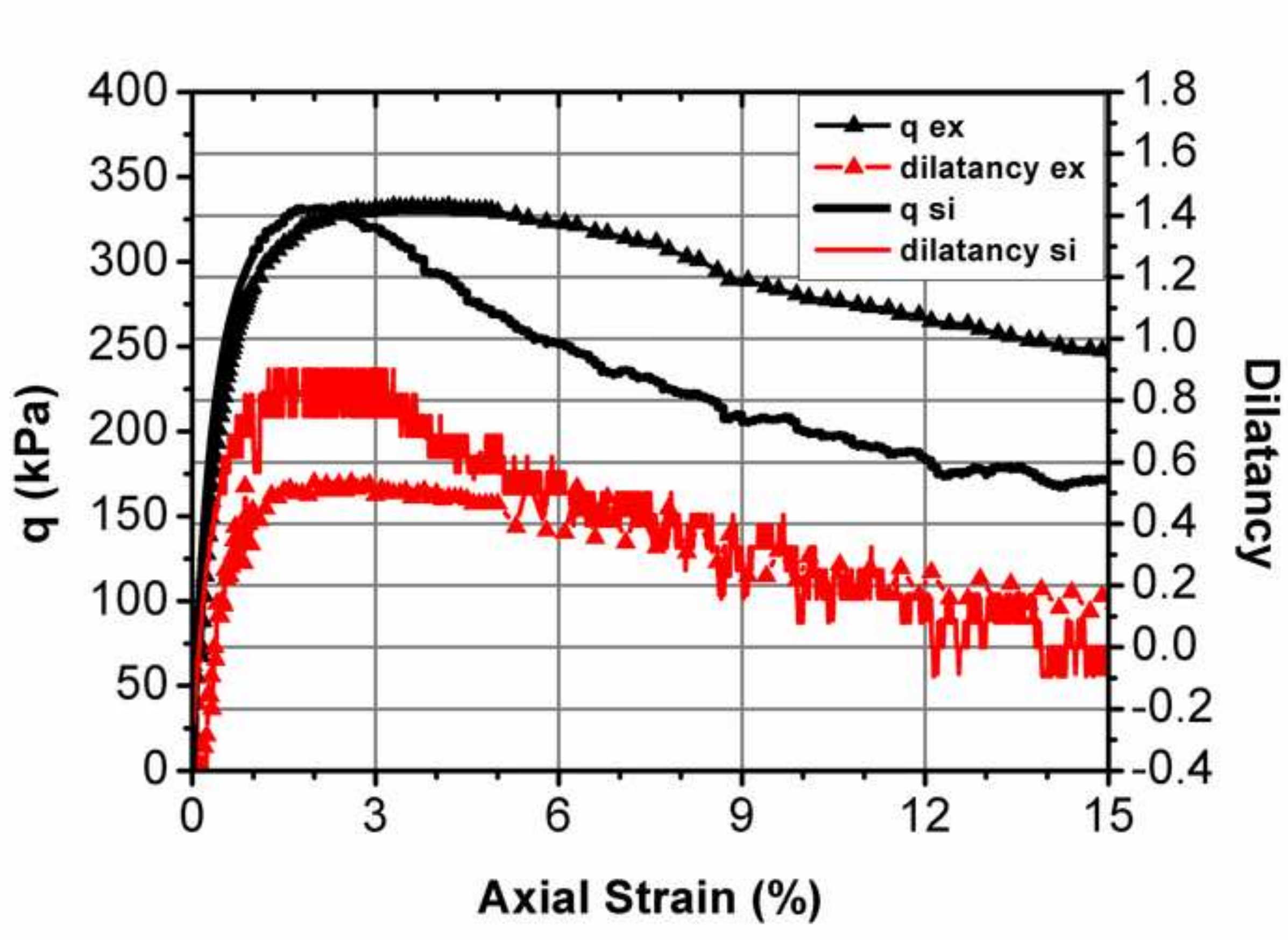


a)

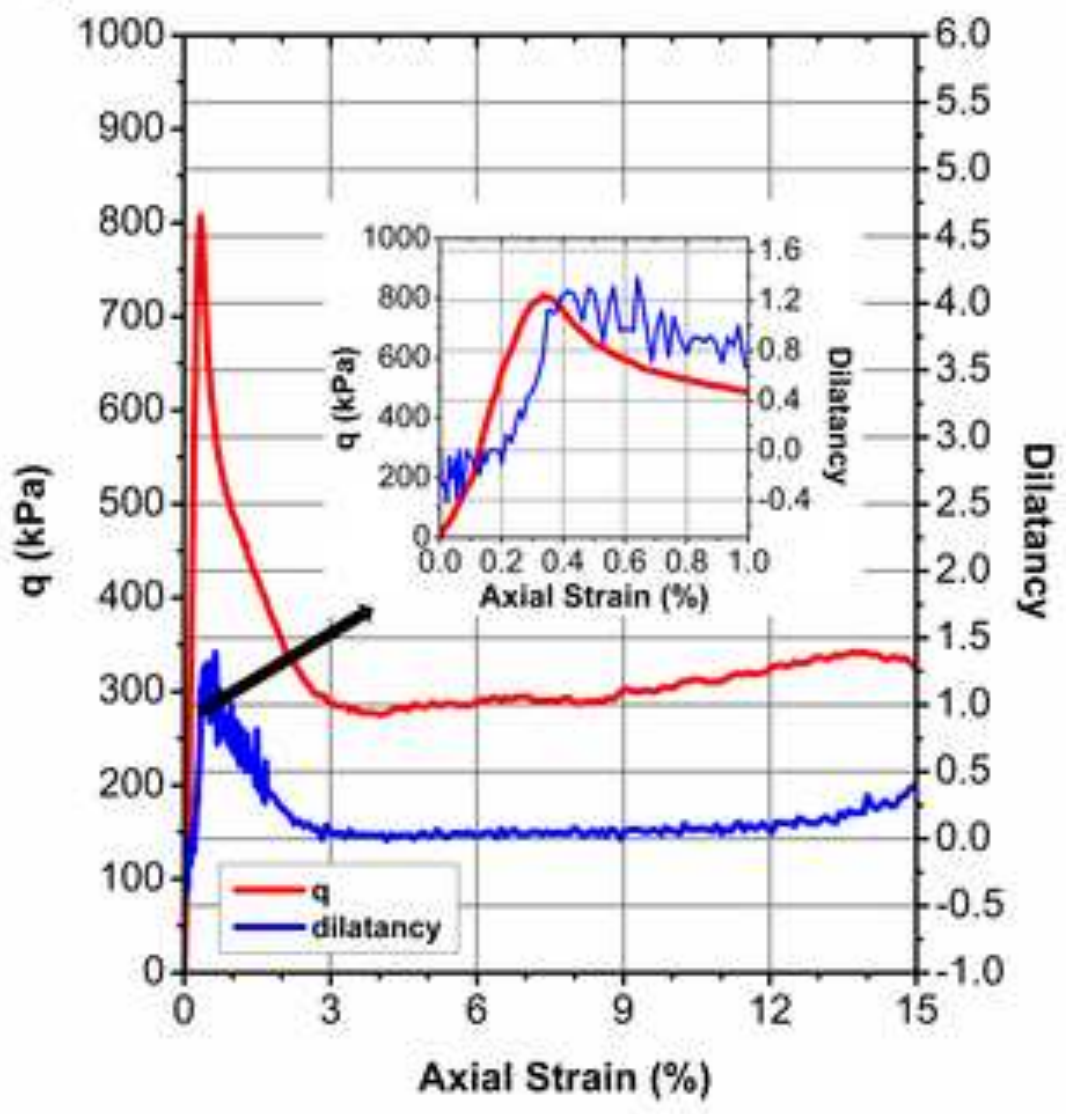

b)

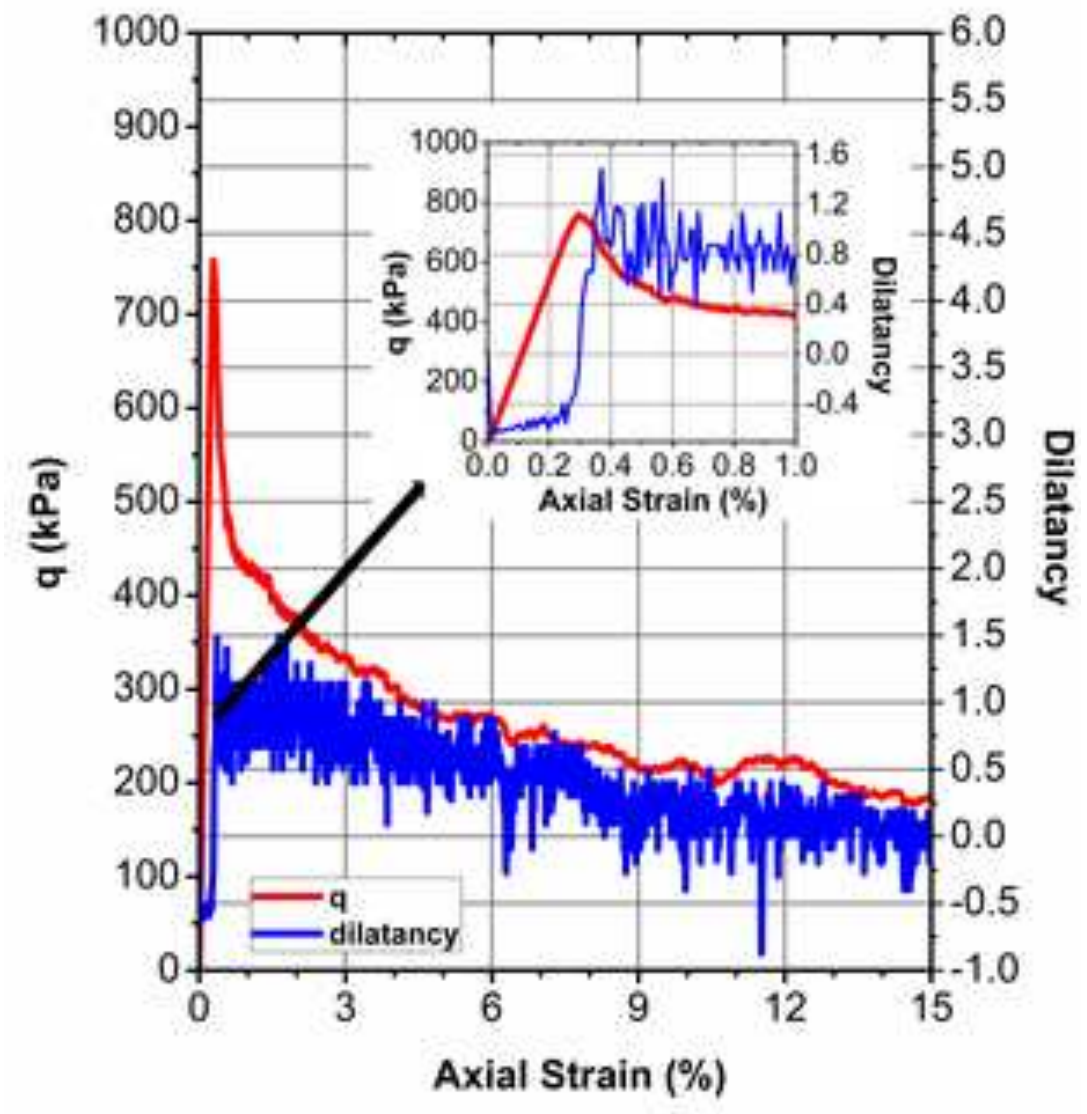

